\title{
CONTAGEM DE FEZES COMO ÍNDICE DE ABUNDÂNCIA DE CAPIVARAS (Hydrochaeris hydrochaeris)
}

\section{GUSTAVO ROMEIRO MAINARDES PINTO}

Dissertação apresentada à Escola Superior de Agricultura "Luiz de Queiroz", Universidade de São Paulo, para obtenção do título de Mestre em Ecologia de Agroecossistemas.

\author{
P I R A C I C A B A \\ Estado de São Paulo - Brasil \\ Setembro - 2003
}




\title{
CONTAGEM DE FEZES COMO ÍNDICE DE ABUNDÂNCIA DE CAPIVARAS (Hydrochaeris hydrochaeris)
}

\author{
GUSTAVO ROMEIRO MAINARDES PINTO \\ Engenheiro Agrônomo
}

Orientador: Prof. Dr. HILTON THADEU ZARATE DO COUTO

Dissertação apresentada à Escola Superior de Agricultura "Luiz de Queiroz", Universidade de São Paulo, para obtenção do título de Mestre em Ecologia de Agroecossistemas.

P I R A C I C A B A

Estado de São Paulo - Brasil

Setembro - 2003 


\section{Dados Internacionais de Catalogação na Publicação (CIP) DIVSÃO DE BIBLIOTECA E DOCUMENTACÃO - ESALQ/USP}

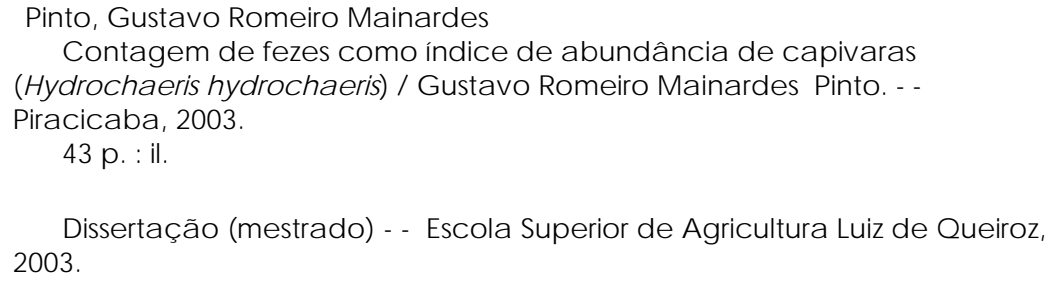

Bibliografia.

1. Capivaras-Monitoramento 2. Fezes 3. Manejo animal I. Título

CDD 636.9323

\section{"Permitida a cópia total ou parcial deste documento, desde que citada a fonte - O autor"}




\section{SUMÁRIO}

Página

RESUMO............................................................................ V

SUMMARY ......................................................................... vii

1 INTRODUÇÃ

2 REVISÃO DE LITERATURA..................................................... 7

3 MATERIAL E MÉTODOS.......................................................... 15

3.1 Levantamento............................................................... 15

3.2 Caracterização das áreas de estudo.......................................... 17

3.3 Condução das coletas de dados.............................................. 19

3.4 Análises dos dados.............................................................. 20

4 RESULTADOS E DISCUSSÃO................................................ 21

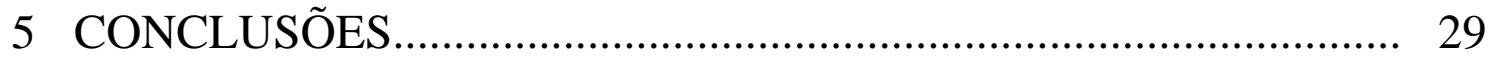

REFERÊNCIAS BIBLIOGRÁFICAS.............................................. 30

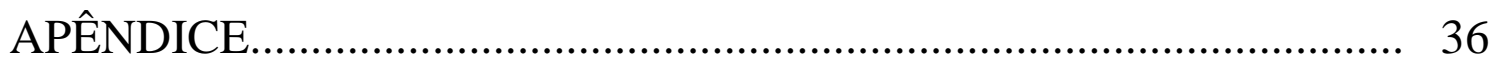




\section{AGRADECIMENTOS}

Este trabalho possui alguns "pais” que foram fundamentais desde sua gestação até sua conclusão. A quem devo muita gratidão:

Sem dúvida, o auxílio da doutoranda Kátia Maria Paschoaletto Michi de Barros Ferraz foi decisivo, tanto em questões intelectuais quanto práticas e até financeiras.

Meus pais Cleide e Sérgio e minha esposa Desirrê deram o apoio moral e material indispensáveis, mesmo nos momentos mais difíceis.

Os professores Hilton Thadeu e Luciano Verdade foram verdadeiramente orientadores.

Agradeço também à Prefeitura do Campus “Luiz de Queiroz”, à empresa Caterpillar e ao setor de Parques e Jardins de Campinas (SP) por cederem seus espaços onde o estudo foi conduzido.

E, por fim, agradeço a Deus e às suas criaturas: homens, capivaras, terra, água, matas...

Muito obrigado a todos vocês! 


\title{
CONTAGEM DE FEZES COMO ÍNDICE DE ABUNDÂNCIA DE CAPIVARAS (Hydrochaeris hydrochaeris)
}

\author{
Autor: GUSTAVO ROMEIRO MAINARDES PINTO \\ Orientador: Prof. Dr. HILTON THADEU ZARATE DO COUTO
}

\section{RESUMO}

A capivara é uma espécie da fauna silvestre que se favoreceu da situação de conversão de áreas florestais para fins agropecuários, tornando-se bastante comum. Tal superpopulação causa conflitos, especialmente em virtude de prejuízos agrícolas e riscos de transmissão de doenças. Entretanto, a espécie apresenta grande potencial zootécnico e econômico, sendo que, aparentemente, o sistema produtivo mais sustentável seria o extensivo. Para fornecer subsídios técnicos a este sistema, torna-se necessário o monitoramento das populações. Um dos métodos mais utilizados para populações de capivaras é o de contagem direta dos animais, utilizada usualmente em ambientes mais abertos, porém pouco eficiente em locais de vegetação mais fechada e para populações mais arredias. O que se buscou testar neste trabalho foi um método de monitoramento alternativo à contagem direta. $\mathrm{O}$ trabalho foi realizado em sete áreas, sendo quatro com populações fechadas e três abertas. Semanalmente foram contadas as fezes depositadas durante esse período numa área amostral, contados diretamente os animais e levantados dados sobre flutuação real das populações fechadas. A obtenção de um índice de 
abundância através da contagem de fezes apresentou um coeficiente de regressão linear melhor que a contagem direta, em comparação com a flutuação real da população, obtida em ambientes fechados. Porém, nenhum dos métodos apresentou resultados satisfatórios que refletissem, no índice, as alterações ocorridas na população. A contagem de fezes mostrou-se satisfatória para separar em faixas de número de indivíduos as diferentes populações e também na aplicação da estimativa populacional baseada na técnica de Índice-manipulação-índice. O trabalho levanta inúmeras questões a respeito da intensidade amostral, de possíveis estudos quanto ao uso do habitat e do grau de confiabilidade dos monitoramentos baseados em contagens diretas. 


\title{
FECES COUNTING AS AN INDEX OF ABUNDANCE OF CAPYBARAS (Hydrochaeris hydrochaeris)
}

\author{
Author: GUSTAVO ROMEIRO MAINARDES PINTO \\ Adviser: Prof. Dr. HILTON THADEU ZARATE DO COUTO
}

\section{SUMMARY}

The capybara is a wild species that would be favored by the conversion of the forest areas to farmlands, becoming abundant. Such overpopulation causes conflicts, especially due to agricultural damages and risks of transmission of illnesses. However, this species presents a great potential in both aspects: zootechnical and economical. As a result, apparently, the most sustainable productive system would be the extensive one. In order to supply technical subsidies to this system, surveying of populations become necessary. One of the survey methods most used in capybara populations is the direct counting that is usually adopted in ecosystems predominantly herbaceous. However, this methodology relies in some problems. It presents low efficiency in dense forested areas as well as in dealing with fearful capybara individuals. Thus, we proposed in this study a surveying method as an alternative to the direct counting one. The work was done on seven areas (four with closed populations and three open). Weekly, the individuals had been counted, as well as the feces deposited in the period on a sample area, besides getting the real data with respect to fluctuation of the closed populations. The attainment of an index of abundance through the feces counting presented a coefficient of linear 
regression better that the direct counting, in comparison with the real fluctuation of the population, gotten in closed environments. However, none of the methods presented satisfactory results that they reflected, in the index, the occurred alterations in the population. The feces counting revealed satisfactory to also separate in bands of number of individuals the different populations and in the application to estimate populations based in the technique of Index-manipulation-index. The work raises innumerable questions about the sampling intensity, possible studies about the home range use and the degree of trustworthiness of the surveys based on direct countings. 


\section{INTRODUÇÃO}

O desequilíbrio populacional da capivara no Estado de São Paulo pode ser entendido pelas características intrínsecas da espécie como a alta capacidade reprodutiva, pouca exigência quanto ao habitat e alimentação generalista, aliadas à própria degradação ambiental que a favorece, ao substituir as matas por culturas e pastagens, colocando alimento em abundância dentro da área de vida da espécie e concorrendo para diminuir drasticamente as populações de seus principais predadores naturais, como os grandes felinos e répteis (Panthera onca, Puma concolor e Eunectes notaeus). Além disso, como seu habitat está necessariamente associado à água, novos habitats foram criados pela construção de açudes de variados tamanhos em praticamente toda propriedade rural e pelo represamento dos principais rios do Estado.

Neste cenário, a população de capivaras apresenta crescimento vigoroso e vem se tornando um sério problema ao causar danos consideráveis à agricultura (Ferraz et al., 2003), além de apresentar risco de transmissão de doenças. Caughley \& Sinclair (1994) afirmam que existe uma densidade mínima tal da espécie hospedeira que conduz à persistência de doenças e parasitas no ambiente. Assim, em condições de superpopulação a incidência desses males tende sempre a aumentar.

A situação de crescimento da população de capivaras no Estado de São Paulo chegou a tal ponto que a colonização de novos ambientes atingiu um dos locais mais inóspitos do País: as margens dos rios Tietê e Pinheiros, dentro da cidade de São Paulo (O Estado de São Paulo, 2002; Tomazela, 2002), como mostra a Figura 1.

A Lei 5.197/67 (Brasil, 1967) proíbe a caça e determina que os animais silvestres são propriedade do Estado. Shaw (1991) afirma que quando a fauna é um 


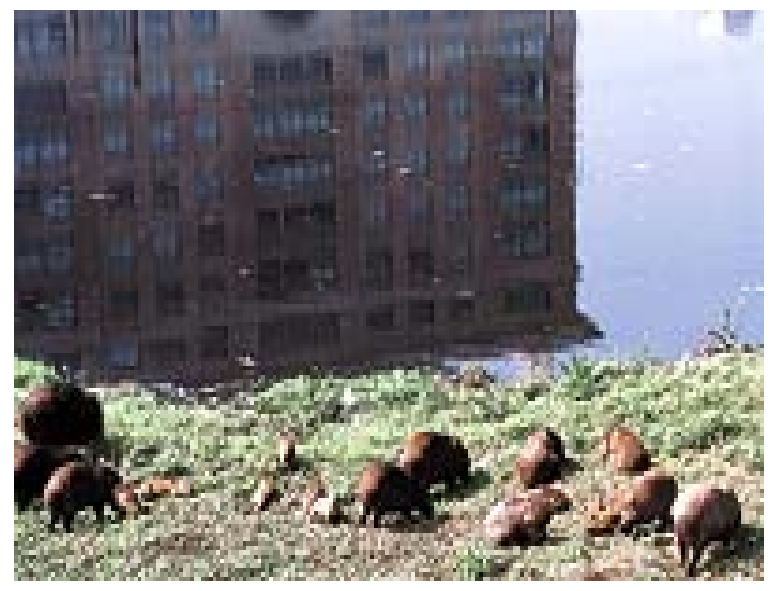

Figura 1 - Capivaras (adultos e filhotes) às margens do rio Pinheiros em São Paulo (SP). Fonte: O Estado de São Paulo

bem comum e é explorada individualmente, tanto legal quanto ilegalmente, quase sempre ocorre uma superexploração desse recurso. Complementando esse raciocínio, Ojasti (1991) conclui que muitos governos latino-americanos esperaram resolver o problema da capivara proibindo toda a caça. Nesse caso, onde houver uma proteção efetiva, a população cresce em poucos anos e demanda algum tipo de manejo; ou acaba controlada naturalmente a uma densidade elevada por competição intraespecífica ou por parasitas e patógenos (Caughley \& Sinclair, 1994). Ainda segundo Ojasti (1991), onde a fiscalização ambiental é deficiente, ocorre o aumento da caça furtiva. Isto é indesejável sob qualquer aspecto, visto que não leva à proteção da espécie, tampouco de seu habitat, causa conflitos e não gera as riquezas que a fauna poderia gerar se explorada racionalmente.

A capivara apresenta grande potencial zootécnico para produção de carne e couro (González-Jiménez, 1995), tendo ainda como sub-produto comercial a gordura subcutânea, produto muito valorizado na medicina popular para o tratamento de asma, bronquite, entre outros (Ojasti, 1991). Apresenta alta eficiência reprodutiva, alimentação de baixo custo e alta taxa de desfrute (Ojasti, 1991), e conta com o fato de que mercados formais para as carnes de animais silvestres vêm crescendo em nosso País (NogueiraFilho, 1996). 
A criação de capivara em cativeiro foi estudada de 1985 a 1998 pelo Centro Interdepartamental de Zootecnia e Biologia de Animais Silvestres (CIZBAS), do Departamento de Zootecnia da Escola Superior de Agricultura “Luiz de Queiroz”, da Universidade de São Paulo (ESALQ/USP), e demonstrou-se ser um animal bastante dócil e de fácil habituação ao contato com o homem, porém de comportamento social bastante complexo, o que dificulta o manejo em cativeiro, que aliado ao custo das instalações e mão-de-obra, aparentemente torna-o inviável economicamente. Some-se ainda o fato de que as criações em cativeiro influem pouco ou nada para a conservação das populações selvagens e principalmente de seus habitats (Freese \& Saavedra, 1991). Assim, sugere-se que o sistema extensivo de manejo seria mais adequado à espécie (Moreira \& MacDonald, 1997).

O Instituto Brasileiro do Meio Ambiente e dos Recursos Naturais Renováveis (IBAMA) é o órgão que atualmente regulamenta o manejo e a exploração econômica de animais silvestres, sendo a Portaria 118-N/1997 que regulamenta os criadouros comerciais. Porém, como não existe uma legislação específica para a capivara, o uso como matrizes dos animais já existentes na propriedade é um tanto quanto nebulosa e a necessidade de marcação e controle individual dos animais no manejo extensivo podem desestimular a criação.

Assim, urge a necessidade de embasamento científico e esclarecimento à opinião pública sobre o uso racional da fauna silvestre, para que os órgãos legisladores e executores das políticas relativas ao meio ambiente possam tomar decisões coerentes. Um bom exemplo de sucesso no manejo extensivo de capivaras que pode nos ajudar na elaboração de planos de manejo é o caso dos Llanos venezuelanos. Ojasti (1991) lista quatro pontos que considera fundamentais para o sucesso do programa venezuelano: (1) É uma atividade tradicional que possui um bom mercado já estabelecido para a carne. (2) É explorada por cada fazendeiro, que pede uma licença ao órgão competente para caçar os animais que estão dentro de suas propriedades, o que faz com que esses proprietários protejam “suas” capivaras contra caçadores furtivos e também valorizem seu habitat. (3) A cota de extração é baseada no monitoramento da população e em estimativas realistas da produção líquida anual do “rebanho”. (4) A agência venezuelana 
de vida silvestre considera a capivara como um recurso natural e tem capacidade técnica para conduzir tal programa.

É evidente que também apresenta alguns problemas, como a cota única de extração anual para todos os fazendeiros, o que certamente não reflete a taxa real de cada caso, e a concentração do manejo nas mãos dos grandes e médios proprietários, não beneficiando os pequenos. Nesse caso a organização em cooperativas dos pequenos proprietários poderia ser uma solução (Ojasti, 1991). Mas o interessante é que houve um planejamento baseado em conhecimentos técnicos que permitiram o sucesso e agora permitem o aperfeiçoamento do sistema.

Outro fator de suma importância é a valorização econômica do habitat natural ocupado pela espécie, que no caso da capivara engloba as matas ciliares e planícies de inundação. Essas áreas têm funções muito importantes na conservação e manutenção da qualidade da água e da biodiversidade, e vêm sendo cada vez mais reconhecidas publicamente por tais atributos, tornando-se evidente que a degradação ambiental está causando prejuízos muito grandes tanto ao abastecimento público de água quanto para o setor energético brasileiro (Novaes, 2001; O Estado de São Paulo, 2001). No caso da biodiversidade, essas áreas são estratégicas como corredores de fluxo gênico entre populações tanto da fauna como da flora, visto que populações isoladas, mesmo em reservas muito bem protegidas, são incapazes de se manter por longos períodos (Shaw, 1991).

Redford \& Robinson (1991) e Ojasti (1973; 1991) apontam alguns dados da importância econômica da capivara na Argentina, Venezuela e Amazônia brasileira, mostrando que mesmo com sistemas produtivos bastante rudimentares, a capivara tratase de um recurso natural renovável com potencial para geração de riquezas.

No caso do Estado de São Paulo, o manejo extensivo poderia aproveitar essas potencialidades de maneira a inverter a situação que se apresenta, ou seja, diminuindo os danos provocados pela superpopulação e gerando renda e empregos, tendo também como vantagem adicional o fato de produzir como forma de proteger também seu habitat natural e a biodiversidade associada a ele (Nogueira-Filho, 1999). Gardner (1991) e Robinson \& Redford (1991) são enfáticos em afirmar que as pessoas usam a fauna 
silvestre, mas geralmente não conseguem valorar essa riqueza e quando a fauna não possui nenhum valor, a própria fauna e seu habitat são destruídos para que a terra sirva a outros usos. Porém os mesmos autores chamam a atenção para o fato de que um uso sustentável não significa somente "usar ou perder”, e sim usar com conhecimento, planejamento e controle.

O manejo racional de fauna silvestre exige a compreensão de inúmeros fatores para que se possam tomar decisões de forma apropriada (Caughley \& Sinclair, 1994), e o conhecimento da biologia da espécie e de um sistema de monitoramento eficiente é a chave fundamental para um bom manejo. A extração pode ser mais ou menos eficiente do ponto-de-vista tanto da conservação quanto da produtividade, dependendo da época, da faixa etária e do sexo dos animais retirados, da porcentagem remanescente da população, entre outros fatores. A taxa de retirada que coloca a população remanescente num nível em que a taxa de crescimento é máxima, é conhecida como "máximo rendimento sustentável”, onde a relação entre o volume de recursos disponível e o número de indivíduos remanescentes gera condições para um aumento exponencial da população (Caughley \& Sinclair, 1994), sendo o objetivo a atingir em uma exploração racional.

De forma geral, os levantamentos populacionais de capivaras têm sido realizados através de contagens diretas dos indivíduos. Porém, em áreas antropizadas e/ou de formação vegetal mais florestal os resultados tendem a subestimar as densidades. Daí a necessidade de utilização de diferentes métodos de levantamentos faunísticos.

A capivara é um animal territorialista, de hábitos regulares, associada a um corpo d’água e que aparentemente não utiliza locais específicos como latrinas, defecando aleatoriamente nos locais onde freqüenta, o que sugere a viabilidade de obtenção de índices de abundância a partir da contagem de montículos de fezes em áreas amostrais fixas e em intervalos conhecidos de tempo (Ferraz ${ }^{1}$; Michi, 1999).

Para verificar a viabilidade de se começar um estudo em maior escala nesses moldes, foi realizado um teste-piloto, que acabou fornecendo algumas noções para a

${ }^{1}$ FERRAZ, K.M.P.M.B. (ESALQ/USP. Laboratório de Ecologia Animal, Piracicaba). Comunicação pessoal, 2002. 
metodologia e apresentou resultados interessantes, sugerindo que os estudos prosseguissem.

O presente trabalho objetivou testar o método indireto de obtenção de índices de abundância como forma de monitoramento de populações de capivaras através da contagem de montículos de fezes, em virtude de sua praticidade de aplicação e baixo custo. 


\section{REVISÃO DE LITERATURA}

A capivara, mamífero pertencente à ordem RODENTIA, subordem CAVIOMORPHA, superfamília CAVIOIDEA, família HYDROCHOERIDAE, subfamília HYDROCHOERINAE, gênero HYDROCHAERIS e espécie HYDROCHAERIS; é um herbívoro semi-aquático que se distribui amplamente por toda a América do Sul a leste dos Andes, indo do Panamá ao Uruguai e noroeste da Argentina e atingindo altitudes de até 1500m (Azcárate, 1980; Eisenberg \& Redford, 1999; Emmons, 1990; Nowak \& Paradiso, 1991; Ojasti, 1973).

A capivara adulta atinge em média $1,5 \mathrm{~m}$ de comprimento, $60 \mathrm{~cm}$ de altura e $50 \mathrm{~kg}$ de peso, podendo chegar até aos 90kg (González-Jiménez, 1995; Moreira \& Macdonald, 1997; Ojasti, 1973). Possui um corpo maciço, onde não aparecem praticamente apêndices, visto que a capivara não possui escroto, sendo que os testículos ficam aderidos internamente ao abdômen, não apresentando dimorfismo sexual. A sexagem só é possível com a exposição manual do órgão genital, que é externado pelo único orifício externo da capivara, que expõe o órgão excretor, urinário e genital (Ojasti, 1973). Ojasti (1973) encontrou uma característica proeminência glandular sobre o focinho de machos, porém a presença de uma glândula sebácea sobre o focinho ocorre em todos os indivíduos, de ambos os sexos, sendo que desenvolve-se mais e torna-se proeminente nos machos dominantes, mas igualmente em fêmeas prenhes, como foi verificado em animais selvagens capturados com fins científicos no Campus da ESALQ/USP em Piracicaba (SP), o que a desclassifica como fator seguro na sexagem de capivaras e sugere que esteja ligada à agressividade do animal.

Como forma de adaptação à água, possui olhos e narinas no mesmo plano da cabeça, o que permite a mínima exposição possível quando dentro d’água. As patas 
possuem membranas que unem os dedos e melhoram a capacidade natatória (Ojasti, 1973). Outra característica que reforça a necessidade da água no habitat da capivara é o fato de possuir glândulas sudoríparas pouco desenvolvidas e muito esparsas, sendo a água e os abrigos sombrios fundamentais para a regulação da temperatura corporal (Pereira et al. $^{2}$ ). Animais submetidos involuntariamente a estresse durante capturas e manejo no Campus da ESALQ/USP, em locais onde não tinham acesso à água apresentaram muito rapidamente a hipertermia, tombando e apresentando respiração ofegante, mas recuperando-se após serem banhados.

A capivara é bastante prolífica, apresentando até dois partos por ano, com média de 4 filhotes por parto tendo uma mortalidade de 10\% até o desmame, para condições de cativeiro (Nogueira-Filho, 1996), e uma média de 1,5 partos ao ano em fêmeas selvagens observados por Ojasti (1973). A gestação dura cerca de 150 dias, ocorrendo nascimentos o ano todo, porém com maior concentração durante o período de chuvas (Alho et al., 1989; Ojasti, 1973). Medidas de eficiência reprodutiva apontam uma produção anual de 0,23kg de peso de filhotes por kg de peso da mãe, enquanto que bovinos nas mesmas características de criação a pasto natural nos Llanos venezuelanos produzem 0,04kg de filhotes/kg de peso da mãe por ano (Ojasti, 1991).

As gramíneas, especialmente as de áreas alagadas são o principal item de sua dieta (Moreira \& Macdonald, 1997), consumindo também aguapés, ciperáceas, entre tantos outros vegetais. É um animal monogástrico e sua capacidade digestiva inicia-se por uma excelente trituração das forragens, passando por um processo de fermentação que ocorre no ceco por populações de microorganismos que vivem em relação de mutualismo com a capivara, digerindo os carboidratos estruturais das plantas (Mendes, 1999). Herrera $^{3}$ refere-se a um comportamento de coprofagia nas capivaras, que seria o consumo de fezes após serem depositadas no chão, porém segundo Mendes (1999) a capivara realiza a cecotrofia, que trata-se da ingestão da cecotrofe após a fermentação microbiana no ceco diretamente da região anal e pelo próprio animal que o produziu,

\footnotetext{
${ }^{2}$ Citados por Moreira \& Macdonald (1997).

${ }^{3}$ Citado por Moreira \& Macdonald (1997).
} 
possuindo alto valor protéico e vitamínico (Mendes, 1999), o que aumenta consideravelmente o aproveitamento dos alimentos e explica sua extraordinária capacidade de transformar volumosos ricos em celulose e hemicelulose em carne.

Ocupa os mais variados tipos de habitat, que compreendem, geralmente, uma área de pastagem, um capão de mata e um corpo d’água (Alho et al., 1989; Moreira \& MacDonald, 1997; Ojasti, 1973), sendo cada porção utilizada para uma atividade específica (Azcárate, 1980; MacDonald, 1981). O padrão de utilização do habitat varia sazonalmente (Alho \& Rondon, 1987) acompanhando a disponibilidade de água, pastos e terras secas (Herrera \& MacDonald, 1989). Porém, a mudança sazonal de utilização do habitat não envolve movimentos migratórios, sendo que raramente se deslocam mais que 300 metros da área central de maior atividade do grupo (Herrera \& MacDonald, 1989). Normalmente sua área de vida varia de 5 a 16 ha aproximadamente e contém todos os tipos de habitat e recursos suficientes para garantir a sua sobrevivência (MacDonald, 1981).

A densidade bruta de capivaras varia de 0,07 a 0,14 ind./ha no Pantanal, Brasil (Alho \& Rondon, 1987; Alho et al., 1989; Schaller \& Crawshaw, 1981) e de 0,003 a 3,600 ind./ha nos Llanos venezuelanos (Cordero \& Ojasti, 1989; Eisenberg et al., 1979; MacDonald, 1981; Ojasti \& Burgos, 1985). Quanto à densidade ecológica, no Pantanal ela variou de 0,01 a 0,69 ind./ha (Alho et al., 1989) e, na Venezuela, variou de 0,10 a 1,30 ind./ha (Eisenberg et al., 1979; Herrera, 1986). Já Ferraz et al. (2001), estudando capivaras em ambiente antrópico no Campus “Luiz de Queiroz”, Piracicaba (SP) encontraram uma densidade ecológica média de 4,11 ind./ha, muito superior à encontrada em outras áreas. Os autores sugerem que a elevada densidade pode ser explicada em função do grau de perturbação da paisagem.

São animais sociais que vivem em grupos variando de 3 a 14 indivíduos em média (Alho \& Rondon, 1987; Alho et al., 1989; Herrera, 1986; Herrera \& MacDonald, 1987; MacDonald, 1981; Schaller \& Crawshaw, 1981). Segundo Ojasti (1973), ocorre variação no tamanho dos grupos em função das flutuações sazonais do recurso água. Na estação seca, os indivíduos se concentram ao redor dos limitados corpos de água, formando grupos maiores do que os encontrados na estação chuvosa. Já Herrera \& 
MacDonald (1987) afirmam que os grupos maiores encontrados na estação seca refletem apenas a combinação de várias unidades sociais independentes. Herrera (1986) e Herrera \& MacDonald (1987) verificaram que, apesar das agregações ocorridas na estação seca, a tolerância espacial não destrói a integridade dos grupos, já que o comportamento territorial continua evidente entre os grupos vizinhos. Assim, o tamanho e a composição se mantêm estáveis por dois anos ou mais. Azcárate (1980) observou que, ao final da estação seca, a abundância de água e alimento influi na dispersão dos grupos, mas não altera o seu tamanho e composição.

Com relação à composição sexual adulta, podem ser encontrados três tipos de grupos de capivaras: (a) grupos mistos, onde a proporção de machos para fêmeas pode variar de 1:1,7 a 1:3,0 (Alho \& Rondon, 1987; Alho et al., 1989; Herrera \& MacDonald, 1987; MacDonald, 1981; Schaller \& Crawshaw, 1981); (b) pequenos grupos de machos; e, (c) machos solitários (Alho \& Rondon, 1987; Herrera, 1986; Lord, 1994; MacDonald, 1981; Schaller \& Crawshaw, 1981). Já os casos de fêmeas solitárias são raros (Herrera, 1986).

As capivaras possuem hábitos diurnos com pico de atividades concentrado nos períodos vespertino e crepuscular. O ritmo diário de suas atividades resume-se basicamente em forrageio nas primeiras horas da manhã e ao anoitecer e repouso e atividades aquáticas nas horas mais quentes do dia, assim, levantamentos populacionais de capivaras têm sido realizados basicamente através de contagens diretas dos indivíduos durante sua atividade de alimentação (Alho et al., 1989; Azcárate, 1980; Cordero \& Ojasti, 1981; Herrera, 1986; Herrera \& MacDonald, 1989; Jorgenson, 1986; MacDonald, 1981; Mourão \& Campos, 1995; Mourão \& Magnusson, 1997; Ojasti, 1973; Schaller \& Crawshaw, 1981). No entanto, a pressão de caça pode alterar o comportamento social das espécies ou a forma como usam o ambiente (Verdade, 1996). Assim, em áreas muito urbanizadas ou onde sofrem forte pressão de predação, as capivaras podem tornar-se ativas somente à noite (Emmons, 1990; Ojasti, 1973). Desta forma, levantamentos populacionais de capivaras em áreas fortemente impactadas pelo homem tendem a ser subestimados. 
Um dos métodos indiretos que vêm sendo usados para a estimativa de densidade e tamanho de populações de vertebrados de médio a grande porte é a contagem de montículos de fezes por unidades de área por período de tempo. Foi utilizado pelos Estados de Michigan, Nevada e Utah, nos Estados Unidos, em seus primeiros levantamentos faunísticos (Bowden et al., 1969; Robinette et al., 1958). Esse método tem se mostrado bastante eficiente, tendo por característica o baixo custo do monitoramento e a utilização de materiais e equipamentos de fácil manuseio.

Tendo como conhecimento básico a produção de fezes por animal por período de tempo, obtém-se a estimativa da densidade (Bennett et al., 1940; Eberhardt \& Van Etten, 1956; Freddy \& Bowden, 1983; Hall, 2001; Neff et al., 1965; Neff, 1968). Mais uma opção, apresentada por Marques et al. (2001), foi a utilização do método das distâncias perpendiculares a um trajeto linear para estimar a densidade de fezes por área. Pode-se ainda ter a obtenção de um índice de abundância (Caughley \& Sinclair, 1994) pela contagem das fezes para estudos de flutuação populacional, dado que pode ser usado para comparações temporais, sem necessidade de se conhecer a produção por animal, ou ainda definir uma relação entre o número de fezes por área e contagens diretas terrestres dos animais de acordo com Verdade ${ }^{4}$ ou ainda a relação entre o número de fezes e contagens aéreas, apresentando essa combinação ótimos resultados, de acordo com Cawley (2001).

A contagem de fezes por trajetos de largura definida tende a ser usada em situações que diminuam os custos, visto que podem ser definidos em traçados que facilitem os trabalhos, mas nestes casos geralmente produzem dados enviesados, pois não possuem boa aleatorização, geralmente passando ao largo de inúmeras variáveis ambientais que assim são ignoradas. Entretanto, para o caso de um índice ser suficiente é perfeitamente aplicável. Quando porém são feitos de forma a contemplar as diferentes variáveis, podem tornar-se menos eficientes do ponto-de-vista operacional, sendo preteridos às parcelas ou quadrantes, como afirma Robinette et al. (1958).

${ }^{4}$ VERDADE, L.M. (ESALQ/USP. Laboratório de Ecologia Animal, Piracicaba). Comunicação pessoal, 2001. 
Batcheler (1975) desenvolveu um delineamento para contagem de fezes, baseado no conceito da "distância do vizinho mais próximo" (Batcheler, 1971; Clark \& Evans, 1954). Nesse método há um trajeto com pontos de amostragem espaçados regularmente e, localizado um montículo de fezes, é anotada a distância do centro deste até o centro do ponto de amostragem e até o centro do segundo montículo mais próximo. Essas distâncias é que, analisadas, darão os valores de densidade de fezes. Para determinar a distância máxima de procura a partir dos pontos de amostragem são feitos testes-pilotos de modo que as medidas sejam obtidas no mínimo em metade dos pontos da amostra. A análise estatística deste método é descrita por Batcheler (1975) e corrigida por Fischer (1979).

Um detalhe que deve ser observado é quanto à existência de variações sazonais na produção de fezes pelos animais. Collins \& Urness (1979) encontraram uma grande variação nas taxas de defecação do alce (Alces alces), sendo até duas vezes maior no verão em relação ao inverno. Isto porém deve ser menos intenso nas regiões tropicais, onde a variação sazonal ocorre mais intensamente em relação à secas e chuvas, não sendo relatados porém casos de hibernação em grandes mamíferos tropicais, relativamente às estiagens.

Outra questão importante é que ao definir a periodicidade das contagens das fezes deve-se levar em conta o tempo de "validade” das fezes em cada situação, visto que decorrido certo tempo torna-se difícil a distinção entre montículos e mesmo na identificação do mesmo. Kufeld (1968) estimou que a duração de fezes para visualização variaram, para condições temperadas, entre dois meses e quatro anos, dependendo das condições climáticas, umidade do local da defecação e tipo de alimento consumido. Nessas mesmas condições, entretanto, o fato de ocorrer a queda de folhas no outono funciona como um marcador natural do período, ocultando as fezes depositadas anteriormente. Nas condições climáticas tropicais essa variação também tende a ser grande entre a estação seca e chuvosa, porém numa escala de tempo menor. A definição desses valores entretanto é algo de simples realização, podendo ser conduzida facilmente junto com trabalhos de monitoramento, definindo localmente essas características. Neff (1968) enfatiza também a necessidade de observar a premissa de definir a priori os 
critérios a serem usados para a diferenciação entre montículos, evitando que o mesmo seja contado como sendo mais de uma unidade ou montículos diferentes, porém sobrepostos, sejam contados como um só. Deve-se também definir um método para iniciar um novo período de contagem, que geralmente é feito pela remoção ou marcação das fezes contadas para evitar recontagem das mesmas.

O uso de parcelas para levantamentos de fezes aliado à amostragem sistemática georreferenciada pode ser usado para se estabelecer o uso do habitat pela população (Collins \& Urness, 1981; Leopold et al., 1984; Loft \& Kie, 1988) podendo-se produzir mapas de densidade e distribuição de fauna silvestre com bons níveis de acurácia, o que comprovou Cawley (2001) calibrando este método com levantamentos aéreos.

Entretanto, deve ser observada uma premissa básica que é a da aleatoriedade de defecação dos animais, o que vai ter tanto mais importância para o nível de acurácia do trabalho quanto menor for sua escala. Portanto, a interpretação das informações obtidas pelas fezes em relação ao uso do habitat têm que ser feitas com cautela. Collins \& Urness (1981) reportam que 30\% das fezes do veado-de-cauda-branca são depositadas enquanto o animal está caminhando, enquanto que essa atividade consome apenas 4\% do tempo do animal.

No caso de capivaras, estes animais possuem a característica de, terminado o repouso, o grupo se dirigir à água e defecar, o que constitui uma área preferencial, porém fora da área amostral, que compreende somente as áreas de terra firme. E entretanto, após este ato o grupo se dirige às áreas de alimentação, havendo uma ligeira dispersão e a partir daí a defecação torna-se aleatória, acontecendo à medida da necessidade individual e independente do local (Ferraz ${ }^{5}$; Michi, 1999), entretanto faltam estudos quantitativos para verificar quão aleatória é esta atividade. Caughley (1977) afirma que contagens de vestígios, comparado às contagens de animais, freqüentemente fornecem um índice de densidade mais acurado. Fuller (1991) testou a contagem de montículos de fezes como índice para o monitoramento de Odocoileus virginianus, afirmando, contudo, em outro trabalho (Fuller, 1992) que tal método não seria eficiente.

${ }^{5}$ FERRAZ, K.M.P.M.B. (ESALQ/USP. Laboratório de Ecologia Animal, Piracicaba). Comunicação pessoal, 2001. 
Porém, White (1992) volta a afirmar que Fuller (1991) apresentou resultados interessantes que devem ser melhor trabalhados, o que nos leva a crer que este método é carente de mais estudos e testes, especialmente em condições tropicais. 


\section{MATERIAL E MÉTODOS}

\subsection{Levantamento}

As áreas escolhidas para o trabalho possuem corpo d’água, capão de mata e área aberta para pastagem, permitem a contagem direta diurna dos animais e possuem ao menos algum trecho do perímetro do corpo d’água ocupado por terra nua ou pavimentada ou ainda grama baixa, que permitem uma fácil visualização das fezes.

Nos perímetros dos corpos d’água das áreas de estudo foram definidos trajetos de 2m de largura e comprimento variável formando a área amostral onde foram contadas as fezes e de onde foram feitas as contagens diretas com auxílio de binóculo. Na figura 2 pode-se visualizar a lagoa da Captação, às margens do rio Piracicaba, onde o trajeto aproveitou a existência de uma estrada de terra contornando a lagoa.

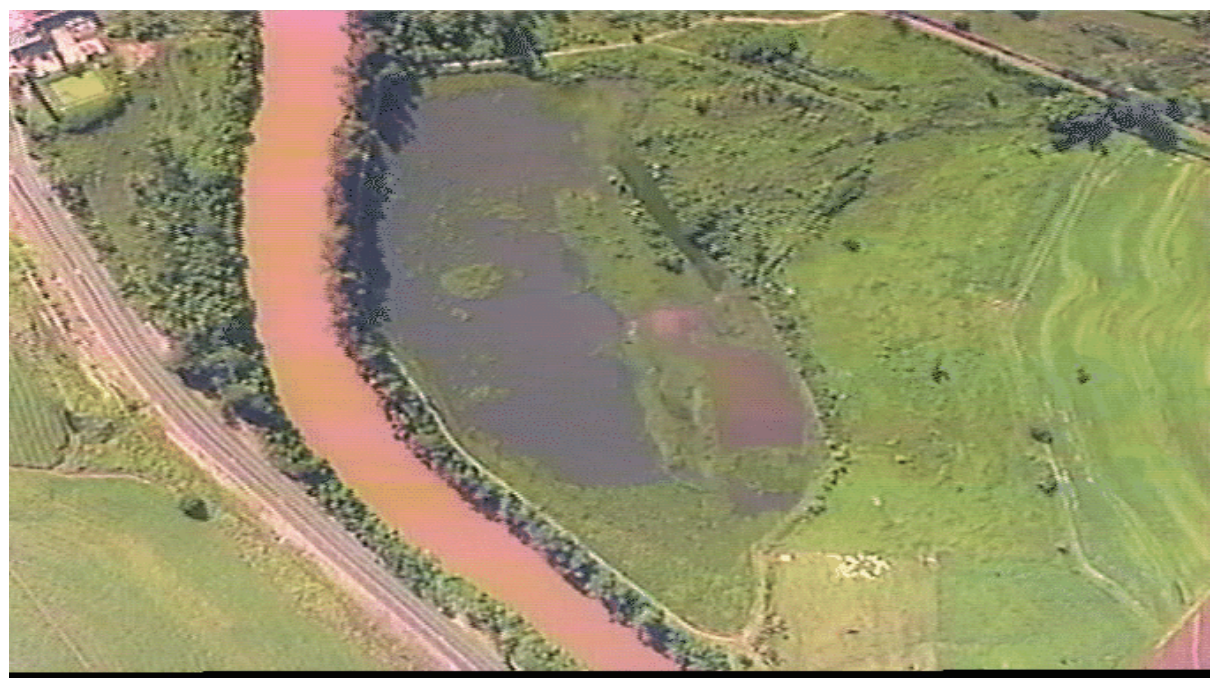

Figura 2 - Vista aérea da lagoa da Captação 
Baseado em observações prévias dos montículos de fezes em levantamentopiloto, definiu-se que os montículos contabilizados teriam que ter, no mínimo, 15 péletes de fezes (Figura 3) (Marques et al., 2001) e que poderia haver uma divisão subjetiva entre adultos e filhotes, dada a clara diferença entre as fezes de ambos. Ainda no mesmo levantamento-piloto, realizado no período de início das chuvas na região, foram marcados com estacas 20 montículos de fezes sobre terra nua e grama para verificação da velocidade de degradação, resultando em fácil visualização de todas mesmo após decorridos os 20 dias, quando foi encerrado o trabalho, o que assegurou que levantamentos semanais poderiam ser feitos sem problemas.

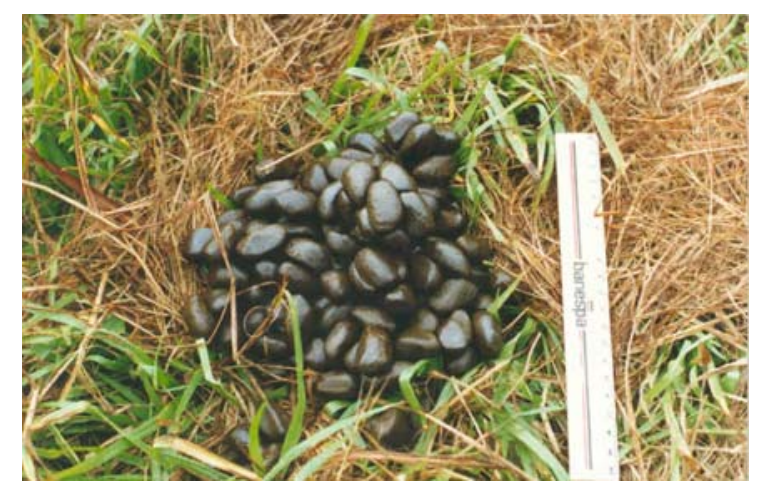

Figura 3 - Fezes de capivara adulta, contada como uma unidade. Podendo-se distinguir claramente os péletes

Foi feita uma primeira remoção total das fezes nas áreas amostrais, iniciando o período de tempo de acúmulo das fezes, sendo essa operação repetida após cada levantamento para que as fezes contadas fossem somente as depositadas durante o intervalo entre um levantamento e outro. Para essa operação primeiramente foi usada uma pá (Figura 4), sendo substituída por um rastelo regulável, o que aumentou a ergonomia e a eficiência do serviço em cerca de 50\%.

Assim, problemas apontados por Sutherland (1996) são eliminados, como grau de persistência das fezes, diferenças de visualização em diferentes habitats e contagem das fezes depositadas realmente em determinado período de tempo. 


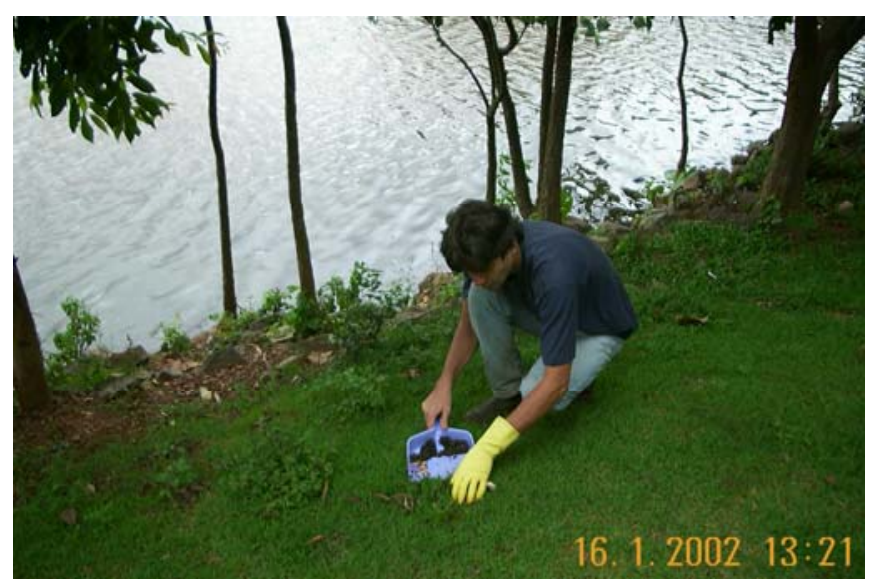

Figura 4 - Contagem e remoção de fezes de capivara na área amostral do lago do Taquaral, Campinas (SP), durante os testes exploratórios

Portanto, em cada trajeto foram feitas simultaneamente duas contagens: o número de animais avistados a partir da área amostral e o número de montículos de fezes depositados dentro da área amostral, sendo ambas as contagens divididas em duas classes de idade: adultos (que no caso deste trabalho incluía também jovens) e filhotes.

Os quatro pontos de coleta de dados localizados no município de Campinas (SP) apresentam a característica de serem parques cercados, onde até mesmo os corpos d’água contam com grades que impedem a entrada e saída de capivaras. Aproveitando esse fato, contando com o auxílio dos responsáveis pela manutenção das áreas, foi possível realizar uma estimativa populacional bastante precisa que forneceu um terceiro dado para essas áreas. Ficando claro que para o índice de abundância, somente a contagem direta a partir da área amostral foi considerada, seguindo-se a metodologia usada para as demais áreas.

\subsection{Caracterização das áreas de estudo}

As áreas de estudo possuem imagens de satélite Landsat 5-TM (situação 1997) obtidas junto ao Projeto Piracena do Centro de Energia Nuclear na Agricultura (CENA) da ESALQ/USP. Através dessas imagens foram obtidas de forma aproximada as áreas e os perímetros dos corpos d’água, utilizando-se o programa Microsoft ArcView 3.2. 
O Campus “Luiz de Queiroz”, da Universidade de São Paulo, localiza-se no município de Piracicaba, Estado de São Paulo. A área total do Campus é de 816,9 ha, sendo 17,0662 ha de área construída e o restante formado por um mosaico de diversas plantações experimentais agrícolas, pastagens e pequenos fragmentos remanescentes de Floresta Atlântica semi-decídua, localizados principalmente nas margens dos rios e açudes, o que reflete de maneira bastante fiel a situação geral das áreas rurais no Estado. Dentro deste, encontra-se duas áreas de coleta de dados:

1) Lagoa de Captação (2242’04.8'’'s 47³6’48.3’’W, 561m de altitude). Açude marginal ao rio Piracicaba, de cerca de 12ha de espelho d'água e 1500m de perímetro, dos quais $1250 \mathrm{~m}$ (83,33\%) foram ocupados pela área amostral de $2500 \mathrm{~m}^{2}$. Esta população vem sendo monitorada semanalmente através de contagens diretas desde julho de 1998 pelo Laboratório de Ecologia Animal da ESALQ/USP. Possui toda sua bacia inserida no Campus e abriga uma boa porção da rica biodiversidade dos ecossistemas alagáveis tropicais, inclusive a mais elevada densidade de capivaras já registrada (Verdade et al. ${ }^{6}$ ).

2) Represa principal da Fazenda Areão da ESALQ/USP (22\%41'51,2’'S 47³3'40'’ W, 540m de altitude), de cerca de 1,8ha de espelho d'água e 500m de perímetro, dos quais $289 \mathrm{~m}$ (52,5\%) foram ocupados pela área amostral de $578 \mathrm{~m}^{2}$. Esta população vem sendo monitorada semanalmente através de contagens diretas desde julho de 1998 pelo Laboratório de Ecologia Animal da ESALQ/USP, sendo que os animais são facilmente avistados devido à habituação ao homem. Informações referentes a mortes e retiradas de animais são repassadas semanalmente pelo funcionário responsável pela fazenda.

3) Açude Unicamp1, com cerca de 4ha de espelho d’água e 1000m de perímetro, dos quais $79 \mathrm{~m}$ (7,9\%) foram ocupados pela área amostral de $158 \mathrm{~m}^{2}$; localizado em área da Funcamp, dentro do Campus da Unicamp (Universidade de Campinas). Coordenadas: $22^{\circ} 48^{\prime} 40.51^{\prime \prime S}$, 4704'01.9"O e 598m de altitude.

\footnotetext{
${ }^{6}$ VERDADE, L.M.; FERRAZ, K.M.P.M.B.; SANTOS-FILHO, R.M.F.; PIFFER, T.R.O. (ESALQ/USP, Piracicaba, SP). Capybara population growth rate and ecological density at carrying capacity on an anthropogenic wetland in southeastern, Brazil. (Em elaboração)
} 
4) Açude Unicamp2, de cerca de 7ha de espelho d’água e $1500 \mathrm{~m}$ de perímetro, dos quais $1100 \mathrm{~m}$ (73,33\%) foram ocupados pela área amostral de $2200 \mathrm{~m}^{2}$; localizado no Parque Municipal de Barão Geraldo. Coordenadas: 2248'35.6"S, 4704'27.1"O e 592m de altitude.

5) Lagoa do Taquaral, de cerca de 14 ha de espelho d’água e $2400 \mathrm{~m}$ de perímetro, dos quais 2200m (91,67\%) foram ocupados pela área amostral de $4400 \mathrm{~m}^{2}$; localizada no Parque Portugal. Coordenadas: 2252'22.0"S, 4702'55.6"O e 595m de altitude.

6) Lagos do Parque do Café, são três lagos muito próximos situados no mesmo curso d’água, com cerca de 3,7ha de espelho d’água e 760m de perímetro, sendo totalmente ocupados pela área amostral de $1520 \mathrm{~m}^{2}$. Coordenadas: 2252'17.0"S, $47^{\circ} 02^{\prime} 51.2^{\prime \prime O}$ e $611 \mathrm{~m}$ de altitude.

7) A última área situa-se dentro do terreno da Caterpillar do Brasil, município de Piracicaba (SP) e contém dois açudes pequenos e próximos cerca de $2 \mathrm{~m}$, um trecho de córrego e uma pequena lagoa natural que compreendem cerca de 0,13ha de espelho d’água e 670m de perímetro, dos quais 192m (28,66\%) foram ocupados pela área

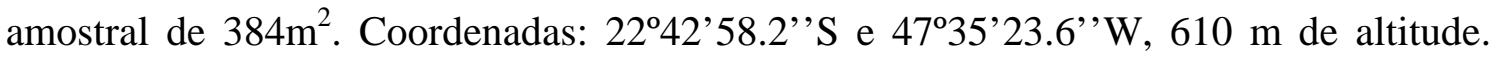
Esta área também foi incluída pela alta densidade de capivaras e pela grande habituação dos animais ao contato humano, permitindo aproximações de até cerca de $2 \mathrm{~m}$ de distância apenas, o que permite uma contagem bastante completa da população.

\subsection{Condução das coletas de dados}

As coletas tiveram periodicidade semanal, sendo anotadas também as condições do tempo. Procurou-se manter sempre o mesmo horário de coleta em cada ponto para diminuir a variabilidade entre os eventos de coleta. Foram coletados os seguintes dados: data, local, hora, contagem direta de adultos e filhotes, contagem de montículos de fezes de adultos e filhotes e a estimativa populacional (para os locais de população fechada).

A cada coleta foi feita também uma média do número de fezes depositadas por dia na área amostral, para padronizar os dados, visto que a periodicidade variava em 
torno de sete dias, sendo porém ocasionalmente menor ou maior, devido a causas operacionais.

\subsection{Análises dos dados}

No teste-piloto os dados foram analisados por correlação de Pearson e regressão linear simples, e extraídas médias simples e erro-padrão (Gomes, 1977).

Os dados do trabalho foram coletados semanalmente e totalizaram 26 coletas a campo para cada um dos sete locais, sendo: contagem direta de capivaras, contagem de fezes/área.tempo e, para as populações fechadas, a estimativa populacional. Todos divididos entre as classes de idade “adultos" e "filhotes".

As contagens e estimativas foram analisadas por regressão linear simples separadamente para cada classe de idade.

Tal desenho pode ser lido como sendo um delineamento inteiramente casualizado, o que permitiu a análise de variância dos resíduos da variável “fezes/área.tempo” e aplicação dos testes de Tukey e Duncan (Gomes, 1977), obtidos através do programa de análises estatísticas Statistical Analysis System (SAS) (SAS INSTITUTE, 1996). 


\section{RESULTADOS E DISCUSSÃO}

Antes do início das coletas oficiais deste estudo, foi feito um teste-piloto visando colher maiores informações e detalhes acerca da metodologia a ser utilizada. Foi feito um trajeto linear no perímetro do corpo d’água da Lagoa da Captação nos mesmos moldes já descritos em Material e Métodos. A Figura 5 corresponde a este teste.
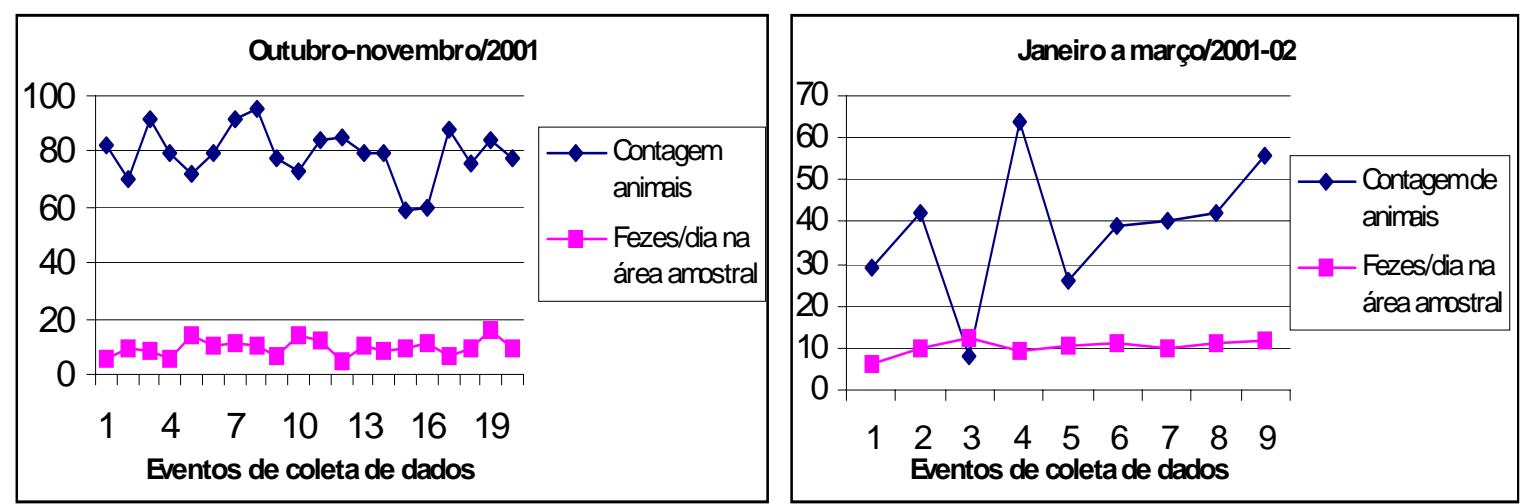

Figura 5 - Levantamentos-piloto realizados para capivaras adultas

Os resultados indicaram que não foram significativas as análises de correlação de Pearson e regressão linear simples entre as contagens diretas e as contagens diárias de montículos de fezes para a classe de animais adultos. Entretanto, analisando-se conjuntamente as classes de adultos e filhotes, como existem claramente duas faixas de abundância bem marcadas, obteve-se uma correlação altamente significativa de 0,8808 e $\mathrm{R}^{2}:$ 0,77 e Prob>F: 0,0001.

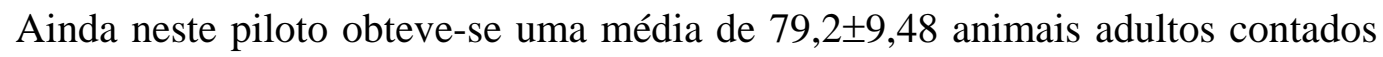
diretamente em outubro/novembro de 2001, com um pico de 100 animais avistados num 
dia, época em que há a melhor visibilidade devido à seca, que reduz o obstáculo representado pela vegetação; enquanto nos meses de janeiro, fevereiro e março de 2001

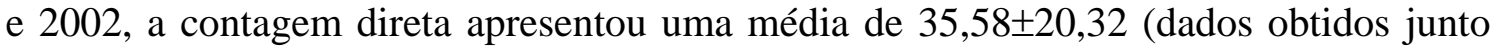
ao Laboratório de Ecologia Animal - ESALQ/USP), portanto com um aumento substancial no erro-padrão. As contagens de fezes, por sua vez, apresentaram valores bastante semelhantes para ambas as estações: média de 38,2+11,48 montículos/ha.dia (em outubro-novembro de 2001) e 38,4£9,2 montículos/ha.dia (janeiro a março/2002), o que sugere que a população pode não ter apresentado uma redução nos níveis que a contagem direta apontou, apesar dos grupos de capivara realizarem dispersões no período de início das chuvas (Azcárate, 1980), e em parte a vegetação mais adensada comprometer as contagens diretas. Diante desses fatos concluiu-se que seria interessante prosseguir o estudo de tal método de monitoramento.

Os resultados, cujos dados são apresentados na planilha de campo (em apêndice), apresentaram correlações não significativas entre contagens diretas, estimativas populacionais (para as populações fechadas) e contagens de montículos de fezes quando as análises de regressão linear simples eram feitas para os diferentes locais e classes de idades separadamente, para qualquer dos pares confrontados, sendo as únicas exceções as correlações entre contagens diretas e estimativas populacionais dos locais Unicamp 2 e Taquaral, cujos dados estão na tabela 1.

Tabela 1. Relações entre contagens diretas de capivaras e estimativas populacionais

\begin{tabular}{ccc}
\hline LOCAIS & CLASSE DE IDADE & COEFICIENTE DE REGRESSÃO LINEAR SIMPLES \\
\hline UNICAMP 2 & ADULTO & $\mathrm{R}^{2}: 0,11$ \\
& FILHOTE & Prob $>\mathrm{F}: 0,0538$ \\
TAQUARAL & ADULTO & $\mathrm{R}^{2}: 0,64$ \\
& & Prob $>\mathrm{F}: 0,0001$ \\
& FILHOTE & $\mathrm{R}^{2}: 0,35$ \\
& & Prob $>\mathrm{F}: 0,0008$ \\
& & $\mathrm{R}^{2}: 0,44$ \\
\hline
\end{tabular}


Esses locais são parques fechados onde os animais são bastante habituados à presença humana, sendo possível uma aproximação de poucos metros, o que facilita a contagem direta. Locais com essas características, entretanto, são exceções à regra geral, especialmente no Estado de São Paulo, onde a vegetação fechada e a maior presença humana tornam os animais bastante furtivos e arredios.

Entretanto, fazendo a análise de regressão aos pares para as contagens diretas, as estimativas populacionais (que foi obtida para as populações dos parques fechados e representada pela própria contagem direta nos locais abertos) e o número padronizado de fezes/hectare.dia com todos os locais conjuntamente para indivíduos adultos, chega-se a resultados bastante interessantes, apresentados na tabela 2.

Tabela 2. Relações entre as estimativas populacionais (populações fechadas), as contagens diretas e de montículos de fezes/hectare.dia

\begin{tabular}{ccc}
\hline PARES RELACIONADOS & CLASSE DE IDADE & COEFICIENTE DE REGRESSÃO LINEAR SIMPLES \\
\hline CONTAGENS DIRETAS & ADULTOS & $\mathrm{R}^{2}: 0,7469$ \\
CONTAGENS DE FEZES & Prob>F: 0,0001 \\
FSTIMATIVAS POPULACIONAIS & ADULTOS & $\mathrm{R}^{2}: 0,0427$ \\
$\mathrm{X}$ & & Prob>F: 0,0118 \\
CONTAGENS DE FEZES & & $\mathrm{R}^{2}: 0,7526$ \\
$\mathrm{~F}$ & Prob>F: 0,0001 \\
$\mathrm{X}$ & & $\mathrm{R}^{2}: 0,033$ \\
ESTIMATIVAS POPULACIONAIS & ADULTOS & Prob>F: 0,0281 \\
CONTAGENS DIRETAS & & $\mathrm{R}^{2}: 0,32$ \\
& FILHOTES & Prob>F: 0,0001 \\
& & $\mathrm{R}^{2}: 0,553$ \\
\hline
\end{tabular}

Por esses resultados pode-se dizer que o índice obtido pelas contagens diretas apresentaram ainda menor correlação com as estimativas populacionais que o obtido pela contagem de fezes. Assim, deve-se ter bem claramente que índices como o das 
contagens diretas podem e devem ser usados, mas geralmente não representam fielmente o que ocorre com a população, especialmente em situações de vegetação mais fechada.

Ambas as contagens não possuem relação direta com as estimativas populacionais quando analisadas numa escala pequena, buscando acusar flutuações dentro de uma população, porém acusam variações entre faixas de número de indivíduos de diferentes populações, portanto, em escala mais ampla, podendo fornecer parâmetros para comparações entre populações para a classe de animais adultos.

No caso da classe de idade de filhotes nenhuma correlação envolvendo as contagens de fezes mostrou-se significativa, podendo ser inferida apenas a presença de filhotes por meio das fezes.

Fazendo-se a análise de variância dos resíduos da variável "montículos de fezes/hectare.dia”, buscando-se verificar quão diferentes faixas de número de indivíduos nas diferentes populações são encontradas, temos os valores mostrados na Tabela 3 abaixo.

Tabela 3. Teste de Tukey $(\mathrm{p}<0,05)$ sobre os resíduos da variável "montículos de fezes/hectare.dia”. Classe de idade adultos. Grupos com a mesma letra não possuem diferença significativa

\begin{tabular}{cccc}
\hline GRUPOS DE TUKEY & MÉDIAS & N & LOCAL \\
\hline A & 76,234 & 26 & AREÃO \\
A & 63,239 & 24 & CATERPILLAR \\
A & 59,498 & 26 & CAPTAÇÃO \\
B & 39,399 & 26 & LAGO DO CAFÉ \\
C & 15,639 & 26 & TAQUARAL \\
C & 15,051 & 26 & UNICAMP 1 \\
C & 3,294 & 26 & UNICAMP 2 \\
\hline
\end{tabular}

O teste de Duncan apresentou os mesmos resultados, sendo que para $\mathrm{p}<0,10$ apenas diferiu significativamente o local Areão em relação aos locais Caterpillar e Captação. 
Verifica-se claramente um problema quanto à área da Fazenda Areão, que conta com uma população contada diretamente média de 8,42 indivíduos, tendo como observação máxima 15 indivíduos adultos e, no entanto, apresentou-se como tendo a maior média. Suspeita-se que o fato de haver uma tela de alambrado contornando a represa pôde contribuir para que a área amostral recebesse a maioria das fezes depositadas no perímetro do corpo d’água, já que a tela encontra-se bastante danificada e uma das principais saídas dos animais fica sobre a área amostral, o que teria causado tal viés nos dados referentes às contagens de fezes. Tal suposição sugere que a contagem de fezes pode ser um bom método auxiliar em estudos de comportamento social e uso do habitat, lançando mão de quadrantes estratificados em forma de ondas, que se iniciariam a partir da manipulação do ambiente no sentido de deixar um único ponto de trânsito obrigatório a partir da saída da água.

Os demais locais apresentaram resultados bastante satisfatórios, como pode-se conferir na Tabela 4 abaixo.

Tabela 4. Médias de contagens diretas, contagens máximas, estimativas populacionais (populações fechadas) e as faixas dos valores do teste de Tukey. Classe de idade adultos.

\begin{tabular}{cccc}
\hline LOCAL & $\begin{array}{c}\text { CONTAGEM DIRETA } \\
\text { MÉDIA E MÁXIMA, } \\
\text { RESPECTIVAMENTE } \\
\left(\mathrm{N}^{\circ} \text { DE INDIVÍDUOS }\right)\end{array}$ & $\begin{array}{c}\text { ESTIMATIVA } \\
\text { POPULACIONAL } \\
\text { MÉDIA DO PERÍODO } \\
\left(\mathrm{N}^{\circ} \text { DE INDIVÍDUOS }\right)\end{array}$ & $\begin{array}{c}\text { FAIXA NO TESTE } \\
\text { DE DUNCAN - } \\
\text { FEZES/HA.DIA }\end{array}$ \\
\hline AREÃO & $8,42-15$ & ----- & $\mathrm{A}$ \\
CATERPILLAR & $24,625-50$ & ---- & $\mathrm{A}$ \\
CAPTAÇÃO & $44,19-67$ & ----- & $\mathrm{A}$ \\
PARQUE DO CAFÉ & $11,46-28$ & 22,23 & $\mathrm{~B}$ \\
TAQUARAL & $8,88-15$ & 12,96 & $\mathrm{C}$ \\
UNICAMP 1 & $4,58-10$ & 7,46 & $\mathrm{C}$ \\
UNICAMP 2 & $5,19-11$ & 8,3 & $\mathrm{C}$ \\
\hline
\end{tabular}


Novos estudos conduzidos com animais sob controle podem gerar respostas interessantes a questões levantadas durante as coletas de dados como a taxa de defecação por animal/dia, quais atividades estão relacionadas com a deposição de fezes e sua quantificação, e mudanças no uso do habitat em decorrência do clima, do estado reprodutivo e da disponibilidade de alimento e sua relação com mudanças na deposição de fezes na área amostral.

Uma sugestão para o uso eficiente deste método de contagem de fezes para a classe de animais adultos pode ser feita por meio da técnica de estimativa populacional descrita por Caughley (1977) como Índice-manipulação-índice, onde um índice de densidade é obtido antes e depois que um número conhecido de animais é adicionado ou removido da população. Em dois dos sete locais estudados houveram capturas durante o período de contagens e que podem fornecer bons resultados, já que nesses locais, por serem parques fechados, a população total é conhecida e respeita-se a premissa de que a precisão da estimativa está ligada ao fato de que a população não sofra migrações durante o experimento.

No caso da lagoa do Taquaral foram capturados 08 adultos e encontrados mais 02 animais mortos, totalizando 10 capivaras adultas retiradas entre os dias 25/04/2002 e 01/06/2002. A média de montículos de fezes por dia na área amostral $\left(4400 \mathrm{~m}^{2}\right)$ nas quatro coletas semanais que antecederam o período de retiradas foi de 9,445. Nas quatro coletas semanais após tal período a média diária de fezes foi de 5,08 montículos. Note-se que esses dados são um índice de densidade, visto que representam fezes depositadas diariamente por área.

Segundo Caughley (1977) temos que:

A estimativa populacional antes da retirada de indivíduos é dada por:

$$
N_{1}=I_{1} \cdot C /\left(I_{2}-I_{1}\right)
$$

e a estimativa populacional após a retirada:

$$
N_{2}=I_{2} \cdot C /\left(I_{2}-I_{1}\right)
$$

sendo que:

$$
\begin{gathered}
I_{1}=\text { índice de densidade anterior às retiradas }=9,445 \text { montículos } \\
I_{2}=\text { índice de densidade após as retiradas }=5,08 \text { montículos }
\end{gathered}
$$


$C=$ número de animais retirados $=-10$ animais

O resultado desta operação aponta para $N_{1}=21,638$ animais adultos e $N_{2}=$ 11,638 animais adultos, sendo que a população conhecida do local era de 20 e 10 animais respectivamente, estando o erro para $N_{1}$ dentro do percentual permissível de $10 \%$, enquanto que $N_{2}$ apresentou erro de 16,38\%. Porém num universo de apenas 10 animais este erro pode ser considerado aceitável, especialmente para esses cálculos, que são bem rígidos.

No caso do Parque do Café foram capturados ou encontrados mortos 18 animais adultos entre os dias 13/03/2002 e 03/04/2002. Porém, havia somente uma semana de coletas anteriores, com média de 5,429 montículos de fezes por dia na área amostral de $1520 \mathrm{~m}^{2}$. Assim, também com apenas uma semana após o período temos 3,8 montículos por dia.

O resultado desta operação apontou $N_{1} \cong 60$ e $N_{2} \cong 42$, enquanto que a população conhecida era de 32 e 15 indivíduos respectivamente, o que sugere que apenas uma coleta com intervalo de uma semana não é suficiente, além do fato de que a área amostral, neste caso, é menor.

O índice de densidade de fezes, portanto, mostra-se como uma boa alternativa para a estimativa populacional de capivaras por meio do Índice-manipulação-índice. Novos estudos devem explorar mais profundamente este assunto, de maneira mais controlada, buscando-se definir métodos de monitoramento aplicados conjuntamente ao manejo adaptativo, para que tais dados construam um sistema que atinja níveis de máximo rendimento sustentável (Ferraz et al., 2001). Tais estudos poderiam, ainda, testar diferentes áreas amostrais para contagem de fezes dentro de cada população, a fim de explorar melhor seu tamanho e localização.

Por fim, durante a realização deste trabalho nos deparamos com a possibilidade de usar os conceitos de metapopulação no intuito de dar prosseguimento ao objetivo mais amplo deste trabalho, que é o manejo extensivo de capivaras e a promoção da conservação de seu habitat. Tais conceitos observam a premissa de que uma população é formada por um agrupamento de populações locais conectadas entre si. A extinção e colonização dessas populações locais, para a população maior, podem ser lidas como 
mortes e nascimentos dentro de uma população local (Hanski \& Simberloff, 1997). Baseando-se nessas premissas surgiram idéias para facilitar a aplicação do manejo extensivo (Ferraz ${ }^{7}$ ). Em estudos de paisagem definiriam-se áreas-núcleo isentas de manejo invasivo, onde a seleção natural estaria sendo garantida e que forneceria excedentes que dispersariam para áreas marginais onde seria feito o manejo com capturas e remoção de animais. Tal sistema praticamente eliminaria os problemas de depreciação estrutural e genética das populações apontados por Ojasti (1991) e causados pela explotação seletiva.

Nestas condições o uso de Índice-manipulação-índice poderia ser uma ferramenta importante na busca pelo máximo rendimento sustentável, que caberia a cada proprietário rural.

7 FERRAZ, K.M.P.M.B. (ESALQ/USP. Laboratório de Ecologia Animal, Piracicaba). Comunicação pessoal, 2002. 


\section{CONCLUSÕES}

Pode-se concluir que este método de monitoramento é uma alternativa interessante para áreas onde os animais são bastante furtivos ou em situações de vegetação bastante fechada, que dificultam ou inviabilizam contagens diretas dos animais, sendo eficiente na diferenciação em faixas de número de indivíduos adultos para comparação entre populações.

Entretanto, deve vir a ser melhor estudado para ser utilizado na técnica de Índicemanipulação-índice (Caughley, 1977), por gerar um índice de densidade. Tal técnica produz uma estimativa populacional, dado de fundamental importância para basear manejos adaptativos.

Os resultados, portanto, são bastante interessantes e necessários como assunto exploratório, apontando para uma alternativa que pode vir a aumentar o leque de opções de métodos de monitoramento, que devem se adequar às diferentes situações.

Neste trabalho, a busca por respostas a um problema (o método de monitoramento) acabou por conduzir também à idealização de novos sistemas de manejo (usando-se os conceitos de metapopulação), tendo como objetivo básico de toda uma equipe de pessoas o manejo extensivo de uma espécie, que torne a praga um recurso econômico obtido de forma sustentável. Diante disso vemos a importância do trabalho em equipe na busca por um objetivo comum. 


\section{REFERÊNCIAS BIBLIOGRÁFICAS}

ALHO, C.J.R.; CAMPOS, V.M.; GONÇALVES, H.C. Ecology, social behavior and management of the capybara in the Pantanal of Brazil. Advances in Neotropical Mammalogy, v.1, p.163-194, 1989.

ALHO, C.J.R.; RONDON, N.L. Habitats, population densities and social structure of capybaras (Hydrochoerus hydrochaeris, Rodentia) in the Pantanal, Brasil. Revista Brasileira de Zoologia, v.4, n.2, p.139-149, 1987.

AZCÁRATE, T. Sociobiologia y manejo del capibara (Hydrochoerus hydrochaeris). Doñana Acta Vertebrata, v.7, n.6, p.228, 1980.

BATCHELER, C.L. Estimation of density from a sample of joint point and nearest neighbour distances. Ecology, v.52, p.703-709, 1971.

BATCHELER, C.L. Development of a distance method for deer census from pellet groups. Journal of Wildlife Management, v.39, p.641-652, 1975.

BENNETT, L.J.; ENGLISH, P.F.; MCCAIN, R. A study of deer populations by use of pellet-group counts. Journal of Wildlife Management, v.4, p.398-403, 1940.

BOWDEN, D.C.; ANDERSON, A.E.; MEDIN, D.E. Frequency distributions of mule deer fecal group counts. Journal of Wildlife Management, v.33, p.895-905, 1969.

BRASIL. Leis, decretos, etc. Lei nº 5.197 de 03 de janeiro de 1967. Diário Oficial, 05 jan. 1967. Seção 1, p. 177: Dispõe sobre a proteção à fauna e dá outras providências.

CAUGHLEY, G. Analysis of vertebrate populations. New York: Wiley-Interscience, 1977. 234p.

CAUGHLEY, G.; SINCLAIR, A.R.E. Wildlife Ecology and Management. Boston: Blackwell Scientific Publications, 1994. 334p. 
CLARK, P.J.; EVANS, F.C. Distance to nearest neighbour as a measure of spatial relationships in populations. Ecology, v.35, n.4, p.445-453. 1954.

COLLINS, W.B.; URNESS, P.J. Elk pellet group distributions and rates of deposition in aspen and lodgepole pine habitats. In: BOYCE, M.S.; HAYDEN-WING, L.D. (Ed.). North American elk: ecology, behavior and management. Laramie: University of Wyoming, 1979. p.140-144.

COLLINS, W.B.; URNESS, P.J. Habitat preferences of mule deer as rated by pelletgroup distributions. Journal of Wildlife Management, v.45, p.969-972, 1981.

CORDERO, G.A.; OJASTI, J. Comparison of capybara populations of open and forested habitats. Journal of Wildlife Management, v.45, n.1, p.267-271, 1989.

EBERHARDT, L.; VAN ETTEN, R.C. Evaluation of the pellet group count as a deer census method. Journal of Wildlife Management, v.20, n.l, p.70-74, 1956.

EISENBERG, J.F.; O’CONNELL, M.A.; AUGUST, P.V. Density, productivity and distribution of mammals in two venezuelan habitats. In: EISENBERG, J.F. (Ed.).Vertebrate ecology in the Northern Neotropics. Washington: Smithsonian Institution Press, 1979. p.187-207.

FERRAZ, K.M.P.M.B.; LECHEVALIER, M.; COUTO, H.T.Z.; VERDADE, L.M. Damage caused by capybaras in a corn field. Scientia Agricola, v.60, n.1, p.191-194, jan./mar. 2003.

FERRAZ, K.M.P.M.B.; SANTOS-FILHO, R.M.F.; PIFFER, T.R.O.; VERDADE, L.M. Biologia e manejo da capivara: do controle de danos ao máximo rendimento sustentável. In: MATTOS, W. R. S. (Ed.) A produção animal na visão dos brasileiros. Piracicaba: FEALQ, 2001. p.580-588.

FISCHER, A.R. Ambiguities in distance method for censusing deer by pellet groups. Journal of Wildlife Management, v.43, p.969-970, 1979.

FREDDY, D.J.; BOWDEN, D.C. Sampling mule deer pellet-group densities in juniperpinyon woodland. Journal of Wildlife Management, v.47, p.476-485, 1983. 
FREESE, C.H.; SAAVEDRA, C.J. Prospects for wildlife management in Latin America and the Caribbean. In: ROBINSON, J.G.; REDFORD, K.H. (Ed.). Neotropical wildlife use and conservation. Chicago: The University of Chicago Press, 1991. p.21-26.

FULLER, T.K. Do pellet counts index white-tailed deer numbers and population change? Journal of Wildlife Management, v.55, p.393-396, 1991.

FULLER, T.K. Do pellet counts index white-tailed deer numbers and population change?: a reply. Journal of Wildlife Management, v.56, p.613, 1992.

GARDNER, A.L. Foreword. In: ROBINSON, J.G.; REDFORD, K.H. (Ed.). Neotropical wildlife use and conservation. Chicago: The University of Chicago Press, 1991. p.2.

GOMES, F.P. Curso de estatística experimental. Piracicaba: ESALQ, 1977. 466p.

GONZÁLEZ-JIMÉNEZ, E. El capybara (Hydrochoerus hydrochaeris) - Estado actual de su producción. Roma: FAO, 1995. 112p.

HALL, J.S. Michigan State University Extension - Wildlife! http://www.msue.msu.edu/wildlife/mood/surveys.htm (03 mar. 2002)

HANSKI, I.; SIMBERLOFF, D. The metapopulation approach, its history, conceptual domain, and application to conservation. Metapopulation Biology, v.4, p.5-26, 1997.

HERRERA, E.A. The behavioural ecology of capybara, Hydrochoerus hydrochaeris. Oxford, 1986. 227p. Thesis (Ph.D.) - University of Oxford.

HERRERA, E.A.; MACDONALD, D.W. Group stability and the structure of a capybara population. Symposia of Zoological Society of London, v.58, p.115-130, 1987.

HERRERA, E.A.; MACDONALD, D.W. Resource utilization and territoriality groupliving capybaras (Hydrochoerus hydrochaeris). Journal of Animal Ecology, v.58, p.667-679, 1989.

JORGENSON, J.P. Notes on the ecology and behavior of capybaras in northeastern Colombia. Vida Silvestre Neotropical, v.1, n.1, p.31-40, 1986.

KUFELD, R.C. Use of paint for marking deer peller groups. Journal of Wildlife Management, v.32, n.3, p.592-596, 1968. 
LEOPOLD, B.D.; KRÂUSMAN, P.R.; HERVERT, J.J. The pellet-group census technique as an indicator of relative habitat use. Wildlife Society Bulletin, v.12, p.325-326, 1984.

LOFT, E.R.; KIE, J.G. Comparison of pellet group and radio triangulation methods for assessing deer habitat use. Journal of Wildlife Management, v.52, p.524-527, 1988.

LORD, D. A descriptive account of capybara behaviour. Studies on Neotropical Fauna and Environment, v.29, p.11-22, 1994.

MACDONALD, D.W. Dwindling resources and the social behaviour of capybara, (Hydrochoerus hydrochaeris) (Mammalia). Journal Zoological of London, v.194, p.371-391, 1981.

MARQUES, F.F.C.; BUCKLAND, S.T.; GOFFIN, D.; DIXON, C.E.; BORCHERS, D.L.; MAYLE, B.A.; PEACE, A.J. Estimating deer abundance from line transect surveys of dung: sika deer in southern Scotland. Journal of Applied Ecology, v.38, p.349-363, 2001.

MENDES, A. Determinação da ocorrência de cecotrofia em capivaras (Hydrochoerus hydrochaeris hydrochaeris L. 1766). Piracicaba, 1999. 73p. Dissertação (Mestrado) Escola Superior de Agricultura “Luiz de Queiroz”, Universidade de São Paulo.

MICHI, K.M.P. Estrutura social da capivara (Hydrochaeris hydrochaeris, Rodentia: Caviomorpha) revelada pelas relações espaciais. São Paulo, 1999. 57p. Dissertação (Mestrado) - Instituto de Psiquiatria, Universidade de São Paulo.

MOREIRA, J.R.; MACDONALD, D.W. Técnicas de manejo de capivaras e outros grandes roedores na Amazônia. In: VALLADARES-PÁDUA, C.; BODMER, R.E.; CULLEN Jr., L. (Ed). Manejo e conservação de vida silvestre no Brasil. Brasília: Sociedade Civil Mamirauá, 1997. p.186-213.

MOURÃO, G.; CAMPOS, Z. Survey of broad-snouted caiman (Caiman latirostris), marsh deer (Blastocerus dichotomus) and capybara (Hydrochaeris hydrochaeris) in the area to be inundated by Porto Primavera dam, Brazil. Biological Conservation, v.73, p.27-31, 1995. 
MOURÃO, G.; MAGNUSSON, W.E. Uso de levantamentos aéreos para o manejo de populações silvestres. In: VALLADARES-PÁDUA, C.; BODMER, R.E.; Manejo e conservação de vida silvestre no Brasil Tropical. Brasília: MCT;CNPq;Sociedade Civil Mamirauá, 1997. p. 23-33.

NEFF, D.J. The pellet group count technique for big game trend, census and distribution: a review. Journal of Wildlife Management. v.32, p.597-614, 1968.

NEFF, D.J.; WAILMO, 0.C.; MORRISON, D.C. A determination of defecation rate for elk. Journal of Wildlife Management. v.29, n.2, p.406-407, 1965.

NOGUEIRA-FILHO, S.L.G. Manual de “criação de capivaras”. Viçosa: Centro de Produções Técnicas, 1996. 50p.

NOGUEIRA-FILHO, S.L.G. Criação de cateto e queixada. Viçosa: Centro de Produções Técnicas, 1999. 50p.

NOVAES, W. A mãe de todas as crises. O Estado de São Paulo, 15 jun. 2001. p.2.

O ESTADO DE SÃO PAULO. O preço da imprevidência. http://www.jt.estadao.com.br/editorias/2001/03/26/editoriais002.html (26 mar. 2001).

O ESTADO DE SÃO PAULO. Capivaras no Pinheiros. http://www.estado.estadao.com.br/editorias/2002/07/27/cid006.html (27 jul. 2002).

OJASTI, J. Estudio biológico del chigüire o capibara. Caracas: Fondo Nacional de Investigaciones Agropecuarias, 1973. 257p.

OJASTI, J. Human exploitation of capybara. In: ROBINSON, J.G.; REDFORD, K.H. (Ed.). Neotropical wildlife use and conservation. Chicago: The University of Chicago Press, 1991. p.236-254.

OJASTI, J.; BURGOS, S. Density regulation in population of capybara. Acta Zoologica Fennica, v.173, p.81-83, 1985.

ROBINETTE, W.L.; FERGUSON, R.B.; GASHWILER, J.S. Problems involved in the use of deer pellet group counts. Transcriptions of North American Wildlife Conference, v.23, p.411-425, 1958.

ROBINSON, J.G.; REDFORD, K.H. The use and conservation of wildlife. In: ROBINSON, J.G.; REDFORD, K.H. (Ed.). Neotropical wildlife use and conservation. Chicago: The University of Chicago Press, 1991. p.3-5. 
SAS INSTITUTE. SAS Sytem Release 6.12. Cary: 1996. 214p.

SCHALLER, G.S.; CRAWSHAW, P.G. Social organization in a capybara population. Saugetierkundliche Mitteilungen, v.29, p.3-16, 1981.

SHAW, J.H. The outlook for sustainable harvests of wildlife in Latin America. In: ROBINSON, J.G.; REDFORD, K.H. (Ed.). Neotropical wildlife use and conservation. Chicago: The University of Chicago Press, 1991. p.24-36.

SUTHERLAND, W.J. Ecological census techniques: a handbook. Cambridge: Cambridge University Press, 1996. 336p.

TOMAZELA, J.M. Equipe captura capivaras no rio Tietê em São Paulo. O Estado de São Paulo, São Paulo, 07 ago. 2002. p.9C.

VERDADE, L.M. The influence of hunting pressure on the social behavior of vertebrates. Revista Brasileira de Biologia, v.56, n.1, p.1-13, 1996.

WHITE, G. C. Do pellet counts index white-tailed deer numbers and population change?: a comment. Journal of Wildlife Management, v.56, n.3, p.611-612, 1992. 


\section{APÊNDICE}


Tabela 1. Planilha de campo

\begin{tabular}{|c|c|c|c|c|c|c|c|c|}
\hline \multirow[t]{2}{*}{ Dia } & \multirow[t]{2}{*}{ Local } & \multirow[t]{2}{*}{$\begin{array}{l}\text { Área } \\
\text { Amostral }\end{array}$} & \multirow[t]{2}{*}{ Hora } & \multicolumn{2}{|c|}{ Contagem Direta } & \multicolumn{2}{|c|}{$\begin{array}{c}\text { Contagem de Fezes } \\
\text { Fezes/Dia }\end{array}$} & \multirow{2}{*}{$\begin{array}{l}\text { Observações: } \\
\text { A-adultos } \\
\text { F-Filhotes } \\
\text { Tempo* }\end{array}$} \\
\hline & & & & Adulto & Filhote & Adulto & Filhote & \\
\hline $16 / 01 / 2002$ & Unicamp1 & $158 m^{2}$ & $10: 00$ & 7 & 1 & Limp & Limp & 2 fezes de adultos recentes \\
\hline \multirow[t]{2}{*}{$25 / 02 / 2002$} & Unicamp1 & $158 \mathrm{~m}^{2}$ & $11: 00$ & 7 & 0 & 9 & 0 & N1; V3; C1 \\
\hline & & & & & & 0.225 & $\mathbf{0}$ & Total estimado: 7A \\
\hline \multirow[t]{2}{*}{ 04/03/2002 } & Unicamp1 & $158 m^{2}$ & $10: 40$ & 7 & 0 & 2 & 0 & N1; V2; C1 \\
\hline & & & & & & 0.286 & $\mathbf{0}$ & Total estimado: 7A \\
\hline \multirow[t]{2}{*}{ 18/03/2002 } & Unicamp1 & $158 m^{2}$ & 10:15 & 7 & 0 & 17 & 0 & N2; V1; C1 \\
\hline & & & & & & 1.214 & $\mathbf{0}$ & Total estimado: 7A \\
\hline \multirow[t]{2}{*}{$25 / 03 / 2002$} & Unicamp1 & $158 m^{2}$ & $10: 10$ & 6 & 0 & 4 & 0 & N4; V1; C2 \\
\hline & & & & & & 0.571 & $\mathbf{0}$ & Total estimado: 7A \\
\hline \multirow[t]{2}{*}{ 03/04/2002 } & Unicamp1 & $158 m^{2}$ & $10: 10$ & 0 & 0 & 3 & 0 & N1; V1; C1 \\
\hline & & & & & & 0.333 & $\mathbf{0}$ & Total estimado: $7 \mathrm{~A}$ \\
\hline \multirow[t]{2}{*}{$08 / 04 / 2002$} & Unicamp1 & $158 m^{2}$ & $10: 30$ & 6 & 0 & 3 & 0 & N1; V1; C1 \\
\hline & & & & & & 0.6 & $\mathbf{0}$ & Total estimado: $7 \mathrm{~A}$; $3 \mathrm{~F}$ \\
\hline \multirow[t]{2}{*}{$17 / 04 / 2002$} & Unicamp1 & $158 m^{2}$ & $10: 10$ & 7 & 0 & 0 & 0 & N1; V1; C1 Informação do \\
\hline & & & & & & $\mathbf{0}$ & $\mathbf{0}$ & $\begin{array}{c}\text { guarda local: Nasceram } 3 \\
\text { filhotes há } \pm 15 \text { dias. } \\
\text { Total estimado: } 7 \text { A; } 3 \text { F }\end{array}$ \\
\hline \multirow{2}{*}{$22 / 04 / 2002$} & Unicamp1 & $158 m^{2}$ & 10:45 & 1 & 1 & 1 & 0 & N1; V2; C1 \\
\hline & & & & & & 0.2 & $\mathbf{0}$ & Total estimado: $7 \mathrm{~A}$; 3F \\
\hline \multirow[t]{2}{*}{$29 / 04 / 2002$} & Unicamp1 & $158 \mathrm{~m}^{2}$ & $11: 25$ & 0 & 0 & 3 & 0 & N1; V1; C1 \\
\hline & & & & & & 0.429 & $\mathbf{0}$ & Total estimado: $7 \mathrm{~A}$; 3F \\
\hline 06/05/2002 & Unicamp1 & $158 m^{2}$ & $10: 10$ & 3 & 0 & 3 & 0 & N2; V1; C1 \\
\hline & & & & & & 0.429 & $\mathbf{0}$ & Total estimado: $7 \mathrm{~A}$; 3F \\
\hline $13 / 05 / 2002$ & Unicamp1 & $158 m^{2}$ & $10: 50$ & 4 & 3 & 1 & 0 & N1; V2; C1 \\
\hline & & & & & & 0.143 & $\mathbf{0}$ & Total estimado: $7 \mathrm{~A}$; 3F \\
\hline $20 / 05 / 2002$ & Unicamp1 & $158 m^{2}$ & $10: 10$ & 7 & 2 & 2 & 0 & N2; V1; C1 \\
\hline & & & & & & 0.286 & $\mathbf{0}$ & Total estimado: $7 \mathrm{~A}$; 3F \\
\hline $27 / 05 / 2002$ & Unicamp1 & $158 m^{2}$ & $10: 25$ & 7 & 1 & 0 & 0 & N1; V1; C1 \\
\hline & & & & & & $\mathbf{0}$ & $\mathbf{0}$ & Total estimado: $7 \mathrm{~A}$; 3F \\
\hline 03/06/2002 & Unicamp1 & $158 m^{2}$ & $11: 15$ & 1 & 0 & 1 & 0 & N1; V2; C1 \\
\hline & & & & & & 0.143 & $\mathbf{0}$ & Total estimado: $7 \mathrm{~A}$; 3F \\
\hline $10 / 06 / 2002$ & Unicamp1 & $158 m^{2}$ & $10: 50$ & 0 & 0 & 2 & 0 & N2; V2; C1 \\
\hline & & & & & & 0.286 & $\mathbf{0}$ & Total estimado: $7 \mathrm{~A}$; 3F \\
\hline $19 / 06 / 2002$ & Unicamp1 & $158 m^{2}$ & $10: 50$ & 3 & 3 & 2 & 0 & N1; V1; C1 \\
\hline & & & & & & 0.222 & $\mathbf{0}$ & Total estimado: $7 \mathrm{~A}$; 3F \\
\hline $25 / 06 / 02$ & Unicamp1 & $158 m^{2}$ & $10: 40$ & 6 & 0 & 1 & 0 & N1; V2; C1 \\
\hline & & & & & & 0.167 & $\mathbf{0}$ & Total estimado: $7 \mathrm{~A} ; 3 \mathrm{~F}$ \\
\hline 01/07/2002 & Unicamp1 & $158 m^{2}$ & $10: 40$ & 3 & 0 & 0 & 0 & N1; V1; C1 \\
\hline & & & & & & $\mathbf{0}$ & $\mathbf{0}$ & Total estimado: $7 \mathrm{~A}$; 3F \\
\hline $10 / 07 / 02$ & Unicamp1 & $158 m^{2}$ & $10: 50$ & 6 & 1 & 1 & 0 & N3; V1; C1 \\
\hline & & & & & & 0.11 & $\mathbf{0}$ & Total estimado: $7 \mathrm{~A}$; 3F \\
\hline $25 / 07 / 02$ & Unicamp1 & $158 m^{2}$ & $10: 50$ & 1 & 0 & 0 & 0 & N1; V1; C1 \\
\hline & & & & & & $\mathbf{0}$ & $\mathbf{0}$ & Total estimado: $7 \mathrm{~A}$; 3F \\
\hline 02/08/02 & Unicamp1 & $158 m^{2}$ & $11: 00$ & 2 & 0 & 0 & 0 & N4; V1; C1 \\
\hline & & & & & & $\mathbf{0}$ & $\mathbf{0}$ & Total estimado: $7 \mathrm{~A}$; 3F \\
\hline 09/08/2002 & Unicamp1 & $158 m^{2}$ & $10: 00$ & 10 & 0 & 2 & 0 & N2; V2; C1 \\
\hline & & & & & & 0.286 & $\mathbf{0}$ & Total estimado: $10 \mathrm{~A}$ \\
\hline $16 / 08 / 2002$ & Unicamp1 & $158 m^{2}$ & $10: 40$ & 8 & 0 & 0 & 0 & N1; V1; C1 \\
\hline & & & & & & $\mathbf{0}$ & $\mathbf{0}$ & Total estimado: $10 \mathrm{~A}$ \\
\hline $23 / 08 / 2002$ & Unicamp1 & $158 m^{2}$ & 10:10 & 1 & 0 & 1 & 0 & N1; V1; C1 \\
\hline & & & & & & 0.143 & $\mathbf{0}$ & Total estimado: $10 \mathrm{~A}$ \\
\hline $30 / 08 / 2002$ & Unicamp1 & $158 m^{2}$ & $11: 20$ & 9 & 0 & 0 & 0 & N4; V1; C1 \\
\hline & & & & & & $\mathbf{0}$ & $\mathbf{0}$ & Total estimado: $10 \mathrm{~A}$ \\
\hline $16 / 01 / 2002$ & Unicamp2 & $2200 \mathrm{~m}^{2}$ & 11:00 & 5 & 0 & Limp & Limp & \\
\hline $25 / 02 / 2002$ & Unicamp 2 & $2200 \mathrm{~m}^{2}$ & $12: 00$ & 3 & 8 & 0 & 0 & N1; V2; C1 \\
\hline & & & & & & $\mathbf{0}$ & $\mathbf{0}$ & Total estimado: $4 \mathrm{~A}$; 7F \\
\hline $04 / 03 / 2002$ & Unicamp2 & $2200 \mathrm{~m}^{2}$ & $11: 40$ & 4 & 7 & 0 & 1 & N1; V2; C1 \\
\hline & & & & & & $\mathbf{0}$ & 0.143 & Total estimado: $4 \mathrm{~A}$; 7F \\
\hline
\end{tabular}


Tabela 1. Planilha de campo

\begin{tabular}{|c|c|c|c|c|c|c|c|c|}
\hline \multirow[t]{2}{*}{ Dia } & \multirow[t]{2}{*}{ Local } & \multirow[t]{2}{*}{$\begin{array}{l}\text { Área } \\
\text { Amostral }\end{array}$} & \multirow[t]{2}{*}{ Hora } & \multicolumn{2}{|c|}{ Contagem Direta } & \multicolumn{2}{|c|}{$\begin{array}{c}\text { Contagem de Fezes } \\
\text { Fezes/Dia }\end{array}$} & \multirow{2}{*}{$\begin{array}{l}\text { Observações: A-Adultos } \\
\begin{array}{ll}\text { Tempo* } & \text { F-Filhotes } \\
\end{array}\end{array}$} \\
\hline & & & & Adulto & Filhote & Adulto & Filhote & \\
\hline \multirow[t]{2}{*}{$18 / 03 / 2002$} & Unicamp2 & $2200 \mathrm{~m}^{2}$ & 11:00 & 4 & 7 & 0 & 26 & N2; V2; C1 \\
\hline & & & & & & $\mathbf{0}$ & 1.857 & Total estimado: $4 \mathrm{~A} ; 7 \mathrm{~F}$ \\
\hline \multirow[t]{2}{*}{$25 / 03 / 2002$} & Unicamp2 & $2200 \mathrm{~m}^{2}$ & $10: 50$ & 4 & 7 & 0 & 17 & N4; V1; C2 \\
\hline & & & & & & $\mathbf{0}$ & 2.429 & Total estimado: $4 \mathrm{~A}$; 7F \\
\hline \multirow[t]{2}{*}{$03 / 04 / 2002$} & Unicamp2 & $2200 \mathrm{~m}^{2}$ & $10: 55$ & 3 & 7 & 0 & 15 & N1; V1; C1 \\
\hline & & & & & & $\mathbf{0}$ & 1.667 & Total estimado: $4 \mathrm{~A} ; 7 \mathrm{~F}$ \\
\hline \multirow[t]{2}{*}{$08 / 04 / 2002$} & Unicamp2 & $2200 \mathrm{~m}^{2}$ & $11: 00$ & 3 & 4 & 0 & 23 & N1; V1; C1 \\
\hline & & & & & & $\mathbf{0}$ & 4.6 & Total estimado: $4 \mathrm{~A}$; 7F \\
\hline \multirow[t]{2}{*}{$17 / 04 / 2002$} & Unicamp2 & $2200 \mathrm{~m}^{2}$ & $10: 30$ & 3 & 7 & 0 & 20 & N1; V1; C1 \\
\hline & & & & & & $\mathbf{0}$ & 2.222 & Total estimado: $4 \mathrm{~A}$; 7F \\
\hline \multirow[t]{2}{*}{$22 / 04 / 2002$} & Unicamp2 & $2200 \mathrm{~m}^{2}$ & 11:15 & 4 & 1 & 0 & 5 & N2; V1; C1 \\
\hline & & & & & & $\mathbf{0}$ & 1 & Total estimado: $4 \mathrm{~A} ; 7 \mathrm{~F}$ \\
\hline \multirow[t]{2}{*}{$29 / 04 / 2002$} & Unicamp2 & $2200 \mathrm{~m}^{2}$ & $11: 50$ & 3 & 0 & 0 & 7 & N1; V2; C1 \\
\hline & & & & & & $\mathbf{0}$ & 1 & Total estimado: $4 \mathrm{~A}$; 7F \\
\hline 06/05/2002 & Unicamp2 & $2200 \mathrm{~m}^{2}$ & $10: 45$ & 3 & 2 & 0 & 5 & N2; V2; C1 \\
\hline & & & & & & $\mathbf{0}$ & 0.714 & Total estimado: 4A; 7F \\
\hline $13 / 05 / 2002$ & Unicamp2 & $2200 \mathrm{~m}^{2}$ & $11: 20$ & 1 & 0 & 4 & 5 & N1; V1; C1 \\
\hline & & & & & & 0.571 & 0.714 & Total estimado: $11 \mathrm{~A}$ \\
\hline $20 / 05 / 2002$ & Unicamp2 & $2200 \mathrm{~m}^{2}$ & $10: 40$ & 11 & 0 & 17 & 0 & N2; V1; C1 \\
\hline & & & & & & 2.429 & $\mathbf{0}$ & Total estimado: $11 \mathrm{~A}$ \\
\hline 27/05/2002 & Unicamp2 & $2200 \mathrm{~m}^{2}$ & $11: 15$ & 11 & 0 & 10 & 0 & N1; V1; C1 \\
\hline & & & & & & 1.429 & $\mathbf{0}$ & Total estimado: $11 \mathrm{~A}$ \\
\hline 03/06/2002 & Unicamp2 & $2200 \mathrm{~m}^{2}$ & $12: 15$ & 11 & 0 & 10 & 0 & N1; V1; C1 \\
\hline & & & & & & 1.429 & $\mathbf{0}$ & Total estimado: $11 \mathrm{~A}$ \\
\hline $10 / 06 / 2002$ & Unicamp2 & $2200 \mathrm{~m}^{2}$ & $11: 40$ & 11 & 0 & 14 & 0 & N3; V2; C1 \\
\hline & & & & & & 2 & $\mathbf{0}$ & Total estimado: $11 \mathrm{~A}$ \\
\hline 19/06/2002 & Unicamp2 & $2200 \mathrm{~m}^{2}$ & $11: 30$ & 9 & 0 & 10 & 0 & N1; V1; C1 \\
\hline & & & & & & 1.111 & $\mathbf{0}$ & Total estimado: $11 \mathrm{~A}$ \\
\hline 25/06/02 & Unicamp2 & $2200 \mathrm{~m}^{2}$ & $11: 30$ & 4 & 0 & 8 & 0 & N1; V2; C1 \\
\hline & & & & & & 1.333 & $\mathbf{0}$ & Total estimado: $11 \mathrm{~A}$ \\
\hline 01/07/2002 & Unicamp2 & $2200 \mathrm{~m}^{2}$ & 11:30 & 8 & 0 & 6 & 0 & N1; V1; C1 \\
\hline & & & & & & 1 & $\mathbf{0}$ & Total estimado: $11 \mathrm{~A}$ \\
\hline $10 / 07 / 02$ & Unicamp2 & $2200 \mathrm{~m}^{2}$ & $11: 40$ & 8 & 0 & 0 & 0 & N3; V1; C1 \\
\hline & & & & & & $\mathbf{0}$ & $\mathbf{0}$ & Total estimado: $11 \mathrm{~A}$ \\
\hline 25/07/02 & Unicamp2 & $2200 \mathrm{~m}^{2}$ & $11: 40$ & 3 & 0 & 16 & 0 & N1; V1; C1 \\
\hline & & & & & & 2.67 & $\mathbf{0}$ & Total estimado: $11 \mathrm{~A}$ \\
\hline 02/08/02 & Unicamp2 & $2200 \mathrm{~m}^{2}$ & $11: 45$ & 0 & 0 & 7 & 0 & N4; V1; C1 \\
\hline & & & & & & 0.87 & $\mathbf{0}$ & Total estimado: $11 \mathrm{~A}$ \\
\hline 09/08/2002 & Únicamp 2 & $2200 \mathrm{~m}^{2}$ & $10: 40$ & 8 & 0 & 13 & 0 & N1; V2; C1 \\
\hline & & & & & & 1.857 & $\mathbf{0}$ & Total estimado: $11 \mathrm{~A}$ \\
\hline $16 / 08 / 2002$ & Unicamp2 & $2200 \mathrm{~m}^{2}$ & $11: 30$ & 10 & 0 & 4 & 0 & N1; V1; C1 \\
\hline & & & & & & 0.571 & $\mathbf{0}$ & Total estimado: 11A \\
\hline 23/08/2002 & Unicamp 2 & $2200 \mathrm{~m}^{2}$ & $11: 00$ & 3 & 0 & 4 & 0 & N1; V1; C1 \\
\hline & & & & & & 0.571 & $\mathbf{0}$ & Total estimado: $11 \mathrm{~A}$ \\
\hline $30 / 08 / 2002$ & Unicamp2 & $2200 \mathrm{~m}^{2}$ & $11: 45$ & 1 & 0 & 7 & 0 & N4; V1; C1 \\
\hline & & & & & & 1 & $\mathbf{0}$ & Total estimado: $11 \mathrm{~A}$ \\
\hline $16 / 01 / 2002$ & Taquaral & $4400 \mathrm{~m}^{2}$ & $13: 00$ & 10 & 2 & Limp & Limp & 2 animais jovens \\
\hline $25 / 02 / 2002$ & Taquaral & $4400 \mathrm{~m}^{2}$ & $14: 30$ & 8 & 2 & 77 & 4 & N1; V2; C1 \\
\hline & & & & & & 1.925 & 0.1 & Total estimado: $16 \mathrm{~A}$; $3 \mathrm{~F}$ \\
\hline 04/03/2002 & Taquaral & $4400 \mathrm{~m}^{2}$ & $15: 00$ & 14 & 3 & 74 & 8 & N1; V1; C1 \\
\hline & & & & & & 10.571 & 1.143 & Total estimado: $16 \mathrm{~A}$; $3 \mathrm{~F}$ \\
\hline $18 / 03 / 2002$ & Taquaral & $4400 \mathrm{~m}^{2}$ & $14: 00$ & 10 & 3 & 82 & 10 & N2; V1; C1 \\
\hline & & & & & & 5.857 & 0.714 & Total estimado: $16 \mathrm{~A}$; 3F \\
\hline $25 / 03 / 2002$ & Taquaral & $4400 \mathrm{~m}^{2}$ & $13: 35$ & 15 & 2 & 43 & 7 & N4; V2; C1 \\
\hline & & & & & & 6.143 & 1 & Total estimado: $17 \mathrm{~A}$; 3F \\
\hline 03/04/2002 & Taquaral & $4400 \mathrm{~m}^{2}$ & $14: 00$ & 8 & 0 & 72 & 7 & N2; V2; C1 \\
\hline & & & & & & 8 & 0.778 & Total estimado: $20 \mathrm{~A} ; 2 \mathrm{~F}$ \\
\hline 08/04/2002 & Taquaral & $4400 \mathrm{~m}^{2}$ & 13:50 & 15 & 2 & 43 & 0 & N2; V2; C1 \\
\hline & & & & & & 8.6 & $\mathbf{0}$ & Total estimado: $20 \mathrm{~A} ; 2 \mathrm{~F}$ \\
\hline
\end{tabular}


Tabela 1. Planilha de campo

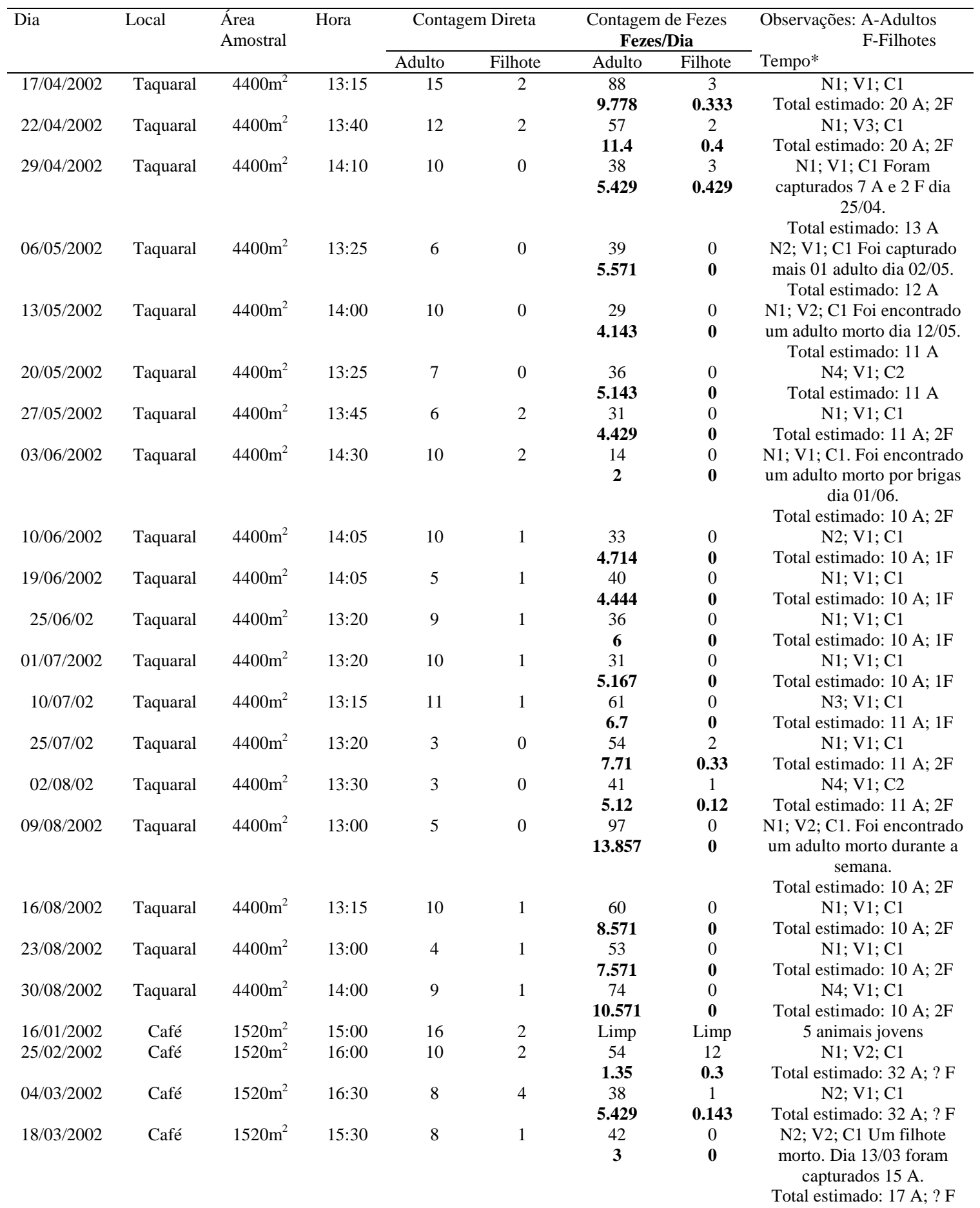


Tabela 1. Planilha de campo

\begin{tabular}{|c|c|c|c|c|c|c|c|c|}
\hline \multirow[t]{2}{*}{ Dia } & \multirow[t]{2}{*}{ Local } & \multirow[t]{2}{*}{$\begin{array}{l}\text { Área } \\
\text { Amostral }\end{array}$} & \multirow[t]{2}{*}{ Hora } & \multicolumn{2}{|c|}{ Contagem Direta } & \multicolumn{2}{|c|}{$\begin{array}{c}\text { Contagem de Fezes } \\
\text { Fezes/Dia }\end{array}$} & \multirow{2}{*}{$\begin{array}{l}\text { Observações: A-Adultos } \\
\\
\text { Tempo* }\end{array}$} \\
\hline & & & & Adulto & Filhote & Adulto & Filhote & \\
\hline $25 / 03 / 2002$ & Café & $1520 \mathrm{~m}^{2}$ & $15: 20$ & 2 & 0 & $\begin{array}{c}26 \\
3.714\end{array}$ & $\begin{array}{l}0 \\
\mathbf{0}\end{array}$ & $\begin{array}{l}\text { N3; V2; C1. As capturas } \\
\text { continuam, sendo que dia } \\
\text { 19/03 foram retirados mais } \\
02 \text { A. } \\
\text { Total estimado: } 15 \text { A; ? F }\end{array}$ \\
\hline 03/04/2002 & Café & $1520 \mathrm{~m}^{2}$ & 16:00 & 9 & 5 & $\begin{array}{c}21 \\
2.333\end{array}$ & $\begin{array}{c}1 \\
\mathbf{0 . 1 1 1}\end{array}$ & $\begin{array}{l}\text { N4; V3; C3. Um adulto } \\
\text { morto. }\end{array}$ \\
\hline 08/04/2002 & Café & $1520 \mathrm{~m}^{2}$ & $15: 20$ & 6 & 3 & $\begin{array}{c}19 \\
3.8\end{array}$ & $\begin{array}{l}0 \\
0\end{array}$ & $\begin{array}{l}\text { Total estimado: } 14 \text { A; } 5 \text { F } \\
\text { N1; V1; C1 } \\
\text { Total estimado: } 14 \text { A; } 5 \text { F }\end{array}$ \\
\hline $17 / 04 / 2002$ & Café & $1520 \mathrm{~m}^{2}$ & $15: 15$ & 8 & 0 & $\begin{array}{c}43 \\
4.778\end{array}$ & $\begin{array}{c}3 \\
0.333\end{array}$ & $\begin{array}{c}\text { N1; V1; C1 } \\
\text { Total estimado: } 14 \text { A; } 5 \text { F }\end{array}$ \\
\hline $22 / 04 / 2002$ & Café & $1520 \mathrm{~m}^{2}$ & $15: 10$ & 7 & 0 & $\begin{array}{l}17 \\
3.4\end{array}$ & $\begin{array}{c}1 \\
0.2\end{array}$ & $\begin{array}{c}\text { N1; V1; C1 } \\
\text { Total estimado: } 14 \text { A; } 5 \text { F }\end{array}$ \\
\hline 29/04/2002 & Café & $1520 \mathrm{~m}^{2}$ & $15: 30$ & 14 & 4 & $\begin{array}{c}32 \\
4.571\end{array}$ & $\begin{array}{l}0 \\
\mathbf{0}\end{array}$ & $\begin{array}{c}\text { N1; V2; C1 } \\
\text { Total estimado: } 14 \text { A; } 5 \text { F }\end{array}$ \\
\hline 06/05/2002 & Café & $1520 \mathrm{~m}^{2}$ & $14: 50$ & 13 & 2 & $\begin{array}{c}26 \\
3.714\end{array}$ & $\begin{array}{c}2 \\
0.286\end{array}$ & $\begin{array}{c}\text { N3; V1; C1 } \\
\text { Total estimado: } 14 \text { A; } 5 \text { F }\end{array}$ \\
\hline $13 / 05 / 2002$ & Café & $1520 \mathrm{~m}^{2}$ & $15: 40$ & 8 & 0 & $\begin{array}{c}24 \\
3.429\end{array}$ & $\begin{array}{l}0 \\
\mathbf{0}\end{array}$ & $\begin{array}{c}\text { N1; V2; C1 } \\
\text { Total estimado: } 18 \text { A; } 2 \text { F }\end{array}$ \\
\hline 20/05/2002 & Café & $1520 \mathrm{~m}^{2}$ & $15: 10$ & 19 & 2 & $\begin{array}{c}48 \\
6.857\end{array}$ & $\begin{array}{c}4 \\
0.571\end{array}$ & $\begin{array}{c}\text { N4; V2; C2 } \\
\text { Total estimado: } 20 \text { A; 4F }\end{array}$ \\
\hline $27 / 05 / 2002$ & Café & $1520 \mathrm{~m}^{2}$ & $15: 10$ & 6 & 1 & $\begin{array}{c}37 \\
5.286\end{array}$ & $\begin{array}{c}5 \\
0.714\end{array}$ & $\begin{array}{c}\text { N1; V1; C1 } \\
\text { Total estimado: } 22 \text { A; } 2 \text { F }\end{array}$ \\
\hline 03/06/2002 & Café & $1520 m^{2}$ & 15:50 & 12 & 0 & $\begin{array}{c}43 \\
6.143\end{array}$ & $\begin{array}{c}2 \\
0.286\end{array}$ & $\begin{array}{l}\text { N1; V1; C1. Foi encontrado } \\
\text { um adulto morto por brigas } \\
\text { dia 01/06. } \\
\text { Total estimado: } 21 \mathrm{~A} ; 2 \mathrm{~F}\end{array}$ \\
\hline 10/06/2002 & Café & $1520 \mathrm{~m}^{2}$ & 15:35 & 22 & 1 & $\begin{array}{c}39 \\
5.571\end{array}$ & $\begin{array}{c}4 \\
0.571\end{array}$ & $\begin{array}{c}\text { N3; V1; C1 } \\
\text { Total estimado: } 22 \text { A; } 1 \mathrm{~F}\end{array}$ \\
\hline 19/06/2002 & Café & $1520 m^{2}$ & 15:50 & 5 & 0 & $\begin{array}{c}28 \\
3.111\end{array}$ & $\begin{array}{l}0 \\
\mathbf{0}\end{array}$ & $\begin{array}{c}\text { N1; V1; C1 } \\
\text { Total estimado: } 22 \text { A; } 1 \mathrm{~F}\end{array}$ \\
\hline 25/06/02 & Café & $1520 \mathrm{~m}^{2}$ & 15:05 & 20 & 0 & $\begin{array}{c}36 \\
6\end{array}$ & $\begin{array}{l}0 \\
\mathbf{0}\end{array}$ & $\begin{array}{c}\text { N1; V1; C1 } \\
\text { Total estimado: } 22 \text { A; } 1 \text { F }\end{array}$ \\
\hline 01/07/2002 & Café & $1520 \mathrm{~m}^{2}$ & 14:50 & 27 & 1 & $\begin{array}{c}58 \\
9.667\end{array}$ & $\begin{array}{c}12 \\
2\end{array}$ & $\begin{array}{c}\text { N1; V1; C1 } \\
\text { Total estimado: } 27 \text { A; } 1 \text { F }\end{array}$ \\
\hline $10 / 07 / 02$ & Café & $1520 m^{2}$ & 14:50 & 28 & 1 & $\begin{array}{r}50 \\
5.5\end{array}$ & $\begin{array}{l}19 \\
2.1\end{array}$ & $\begin{array}{c}\text { N3; V2; C1 } \\
\text { Total estimado: } 28 \text { A; } 1 F\end{array}$ \\
\hline $25 / 07 / 02$ & Café & $1520 m^{2}$ & 14:45 & 10 & 2 & $\begin{array}{c}72 \\
12.0\end{array}$ & $\begin{array}{l}15 \\
2.5\end{array}$ & $\begin{array}{c}\text { N1; V1; C1 } \\
\text { Total estimado: } 28 \text { A; } 1 \mathrm{~F}\end{array}$ \\
\hline $02 / 08 / 02$ & Café & $1520 \mathrm{~m}^{2}$ & 14:55 & 15 & 2 & $\begin{array}{c}75 \\
9.37\end{array}$ & $\begin{array}{l}12 \\
1.5\end{array}$ & $\begin{array}{c}\text { N4; V1; C1 } \\
\text { Total estimado: } 28 \text { A; } 1 F\end{array}$ \\
\hline 09/08/2002 & Café & $1520 \mathrm{~m}^{2}$ & 14:00 & 4 & 0 & $\begin{array}{c}78 \\
11.143\end{array}$ & $\begin{array}{c}1 \\
0.143\end{array}$ & $\begin{array}{c}\text { N1; V2; C1 } \\
\text { Total estimado: } 28 \text { A; 1F }\end{array}$ \\
\hline 16/08/2002 & Café & $1520 \mathrm{~m}^{2}$ & $14: 30$ & 18 & 0 & $\begin{array}{c}54 \\
7.714\end{array}$ & $\begin{array}{l}0 \\
\mathbf{0}\end{array}$ & $\begin{array}{c}\text { N1; V1; C1 } \\
\text { Total estimado: } 28 \text { A; } 1 F\end{array}$ \\
\hline 23/08/2002 & Café & $1520 \mathrm{~m}^{2}$ & $14: 30$ & 6 & 0 & $\begin{array}{c}44 \\
6.286\end{array}$ & $\begin{array}{c}3 \\
0.429\end{array}$ & $\begin{array}{l}\text { N1; V1; C1 } \\
\text { Total estimado: } 28 \text { A; } 1 F\end{array}$ \\
\hline 30/08/2002 & Café & $1520 \mathrm{~m}^{2}$ & 15:00 & 6 & 0 & $\begin{array}{c}87 \\
12.429\end{array}$ & $\begin{array}{l}0 \\
\mathbf{0}\end{array}$ & $\begin{array}{l}\text { N1; V1; C1 } \\
\text { Total estimado: } 28 \text { A; 1F }\end{array}$ \\
\hline $20 / 02 / 2002$ & Captação & $2500 \mathrm{~m}^{2}$ & 16:00 & 39 & 10 & Limp & Limp & 8 jovens \\
\hline $27 / 02 / 2002$ & Captação & $2500 \mathrm{~m}^{2}$ & 15:50 & 29 & 8 & $\begin{array}{c}45 \\
6.429\end{array}$ & $\begin{array}{l}0 \\
\mathbf{0}\end{array}$ & 3 jovens. N4; V1; C2 \\
\hline 06/03/2002 & Captação & $2500 \mathrm{~m}^{2}$ & 16:20 & 42 & 0 & $\begin{array}{l}70 \\
\mathbf{1 0}\end{array}$ & $\begin{array}{c}1 \\
0.143\end{array}$ & 6 jovens N1; V1; C1 \\
\hline $13 / 03 / 2002$ & Captação & $2500 \mathrm{~m}^{2}$ & 16:10 & 8 & 4 & $\begin{array}{c}87 \\
12.429\end{array}$ & $\begin{array}{c}4 \\
0.571\end{array}$ & $\begin{array}{l}\text { N2; V1; C1 Calor muito } \\
\text { intenso }\end{array}$ \\
\hline 20/03/2002 & Captação & $2500 \mathrm{~m}^{2}$ & 16:20 & 64 & 3 & $\begin{array}{c}66 \\
9.429\end{array}$ & $\begin{array}{l}0 \\
\mathbf{0}\end{array}$ & N2; V1; C1 , 23 Jovens \\
\hline
\end{tabular}


Tabela 1. Planilha de campo

\begin{tabular}{|c|c|c|c|c|c|c|c|c|}
\hline \multirow[t]{2}{*}{ Dia } & \multirow[t]{2}{*}{ Local } & \multirow[t]{2}{*}{$\begin{array}{l}\text { Área } \\
\text { Amostral }\end{array}$} & \multirow[t]{2}{*}{ Hora } & \multicolumn{2}{|c|}{ Contagem Direta } & \multicolumn{2}{|c|}{$\begin{array}{c}\text { Contagem de Fezes } \\
\text { Fezes/Dia } \\
\end{array}$} & \multirow{2}{*}{$\begin{array}{l}\text { Observações: A-Adultos } \\
\begin{array}{ll}\text { Tempo* } & \text { F-Filhotes }\end{array} \\
\end{array}$} \\
\hline & & & & Adulto & Filhote & Adulto & Filhote & \\
\hline \multirow[t]{2}{*}{$26 / 03 / 2002$} & Captação & $2500 \mathrm{~m}^{2}$ & $16: 25$ & 26 & 0 & 65 & 0 & N1; V1; C1. 5 Jovens \\
\hline & & & & & & 10.833 & $\mathbf{0}$ & \\
\hline \multirow[t]{2}{*}{$04 / 04 / 2002$} & Captação & $2500 \mathrm{~m}^{2}$ & $16: 15$ & 39 & 5 & 101 & 1 & N1; V1; C1 \\
\hline & & & & & & 11.222 & 0.111 & \\
\hline \multirow[t]{2}{*}{$11 / 04 / 2002$} & Captação & $2500 \mathrm{~m}^{2}$ & $15: 35$ & 40 & 6 & 71 & 1 & N2; V1; C1. 3 Jovens \\
\hline & & & & & & 10.143 & 0.143 & \\
\hline \multirow[t]{2}{*}{$19 / 04 / 2002$} & Captação & $2500 \mathrm{~m}^{2}$ & $15: 30$ & 42 & 9 & 88 & 0 & N1; V1; C1 \\
\hline & & & & & & 11 & $\mathbf{0}$ & \\
\hline \multirow[t]{2}{*}{$25 / 04 / 2002$} & Captação & $2500 \mathrm{~m}^{2}$ & $16: 15$ & 56 & 3 & 72 & 3 & N1; V1; C1. 12 Jovens \\
\hline & & & & & & 12 & 0.5 & \\
\hline \multirow{2}{*}{$02 / 05 / 2002$} & Captação & $2500 \mathrm{~m}^{2}$ & $15: 20$ & 42 & 4 & 103 & 1 & N1; V1; C1. 9 Jovens \\
\hline & & & & & & 14.714 & 0.143 & \\
\hline \multirow[t]{2}{*}{ 09/05/2002 } & Captação & $2500 \mathrm{~m}^{2}$ & $15: 00$ & 41 & 5 & 72 & 0 & N3; V1; C1. 8 Jovens \\
\hline & & & & & & 10.286 & $\mathbf{0}$ & \\
\hline \multirow[t]{2}{*}{$16 / 05 / 2002$} & Captação & $2500 \mathrm{~m}^{2}$ & $15: 00$ & 28 & 11 & 83 & 4 & N4; V2; C1. 7 Jovens \\
\hline & & & & & & 11.857 & 0.571 & \\
\hline $23 / 05 / 2002$ & Captação & $2500 \mathrm{~m}^{2}$ & $15: 10$ & 48 & 11 & 47 & 1 & N1; V3; C1. 2 Jovens \\
\hline & & & & & & 6.714 & 0.143 & \\
\hline 29/05/2002 & Captação & $2500 \mathrm{~m}^{2}$ & $15: 10$ & 50 & 3 & 147 & 6 & N2; V1; C1. 9 Jovens \\
\hline & & & & & & 24.5 & 1 & \\
\hline 06/06/2002 & Captação & $2500 \mathrm{~m}^{2}$ & $14: 55$ & 43 & 5 & 133 & 2 & N2; V1; C1. 5 Jovens \\
\hline & & & & & & 16.625 & 0.25 & \\
\hline 13/06/2002 & Captação & $2500 \mathrm{~m}^{2}$ & $14: 50$ & 43 & 9 & 146 & 6 & N2; V1; C1. 5 Jovens \\
\hline & & & & & & 20.857 & 0.857 & \\
\hline $21 / 06 / 2002$ & Captação & $2500 \mathrm{~m}^{2}$ & $15: 00$ & 50 & 7 & 146 & 6 & N1; V1; C1. 11 Jovens \\
\hline & & & & & & 18.25 & 0.75 & \\
\hline 27/06/02 & Captação & $2500 \mathrm{~m}^{2}$ & $15: 30$ & 58 & 10 & 124 & 2 & N1; V2; C1. 5 Jovens \\
\hline & & & & & & 20.667 & 0.333 & \\
\hline 04/07/2002 & Captação & $2500 \mathrm{~m}^{2}$ & $15: 20$ & 67 & 12 & 80 & 2 & N3; V1; C1. 5 Jovens \\
\hline & & & & & & 11.429 & 0.286 & \\
\hline $11 / 07 / 02$ & Captação & $2500 \mathrm{~m}^{2}$ & $15: 20$ & 36 & 10 & 164 & 6 & N4; V1; C2. 5 Jovens \\
\hline & & & & & & 23.4 & 0.85 & \\
\hline 23/07/02 & Captação & $2500 \mathrm{~m}^{2}$ & $15: 20$ & 60 & 17 & 154 & 4 & N1; V1; C1. 5 Jovens \\
\hline & & & & & & 30.8 & 0.8 & \\
\hline $31 / 07 / 02$ & Captação & $2500 \mathrm{~m}^{2}$ & $14: 55$ & 45 & 14 & 160 & 3 & N4; V3; C3. 5 Jovens \\
\hline & & & & & & 26.67 & 0.5 & \\
\hline 07/08/2002 & Captação & $2500 \mathrm{~m}^{2}$ & $15: 00$ & 58 & 5 & 63 & 1 & N1; V1; C1. 10 Jovens \\
\hline & & & & & & 9 & 0.143 & \\
\hline 13/08/2002 & Captação & $2500 \mathrm{~m}^{2}$ & $15: 10$ & 24 & 0 & 81 & 3 & N1; V1; C1. 1 Jovem \\
\hline & & & & & & 13.5 & 0.5 & \\
\hline 20/08/2002 & Captação & $2500 \mathrm{~m}^{2}$ & $15: 00$ & 48 & 16 & 97 & 0 & N1; V1; C1. 1 Jovem \\
\hline & & & & & & 13.857 & $\mathbf{0}$ & \\
\hline 28/08/2002 & Captação & $2500 \mathrm{~m}^{2}$ & $15: 30$ & 62 & 8 & 161 & 0 & N2; V1; C1. 2 Jovens \\
\hline & & & & & & 20.125 & $\mathbf{0}$ & \\
\hline 20/02/2002 & Areão & $578 \mathrm{~m}^{2}$ & $17: 25$ & 14 & 1 & Limp & Limp & 3 jovens \\
\hline $27 / 02 / 2002$ & Areão & $578 \mathrm{~m}^{2}$ & $17: 40$ & 14 & 0 & 13 & 1 & N4; V1; C2 \\
\hline & & & & & & 1.857 & 0.143 & \\
\hline 06/03/2002 & Areão & $578 m^{2}$ & $17: 50$ & 14 & 1 & 8 & 1 & N2; V1; C1 \\
\hline & & & & & & 1.143 & 0.143 & \\
\hline $13 / 03 / 2002$ & Areão & $578 m^{2}$ & 18:00 & 6 & 0 & 14 & 0 & N2; V2; C1 Calor muito \\
\hline & & & & & & 2 & $\mathbf{0}$ & intenso \\
\hline $20 / 03 / 2002$ & Areão & $578 \mathrm{~m}^{2}$ & 18:00 & 15 & 0 & 39 & 0 & N2; V1; C1, 8 Jovens \\
\hline & & & & & & 5.571 & $\mathbf{0}$ & \\
\hline 26/03/2002 & Areão & $578 m^{2}$ & 18:00 & 15 & 0 & 23 & 0 & N1; V1; C1 \\
\hline & & & & & & 3.833 & $\mathbf{0}$ & \\
\hline 04/04/2002 & Areão & $578 \mathrm{~m}^{2}$ & 18:10 & 15 & 0 & 38 & 0 & N1; V2; C1 \\
\hline & & & & & & 4.222 & $\mathbf{0}$ & \\
\hline
\end{tabular}


Tabela 1. Planilha de campo

\begin{tabular}{|c|c|c|c|c|c|c|c|c|}
\hline \multirow[t]{2}{*}{ Dia } & \multirow[t]{2}{*}{ Local } & \multirow[t]{2}{*}{$\begin{array}{l}\text { Área } \\
\text { Amostral }\end{array}$} & \multirow[t]{2}{*}{ Hora } & \multicolumn{2}{|c|}{ Contagem Direta } & \multicolumn{2}{|c|}{$\begin{array}{c}\text { Contagem de Fezes } \\
\text { Fezes/Dia }\end{array}$} & \multirow{2}{*}{$\begin{array}{l}\text { Observações: A-Adultos } \\
\\
\text { Tempo* }\end{array}$} \\
\hline & & & & Adulto & Filhote & Adulto & Filhote & \\
\hline \multirow[t]{2}{*}{$11 / 04 / 2002$} & Areão & $578 m^{2}$ & $17: 45$ & 15 & 0 & 53 & 0 & N1; V1; C1 \\
\hline & & & & & & 7.571 & $\mathbf{0}$ & \\
\hline \multirow[t]{2}{*}{$19 / 04 / 2002$} & Areão & $578 m^{2}$ & $17: 45$ & 15 & 0 & 50 & 0 & N1; V1; C1 \\
\hline & & & & & & 6.25 & $\mathbf{0}$ & \\
\hline \multirow[t]{2}{*}{$25 / 04 / 2002$} & Areão & $578 m^{2}$ & $15: 25$ & 6 & 0 & 36 & 0 & N1; V1; C1 \\
\hline & & & & & & 6 & $\mathbf{0}$ & \\
\hline \multirow[t]{2}{*}{$02 / 05 / 2002$} & Areão & $578 \mathrm{~m}^{2}$ & $17: 40$ & 10 & 0 & 51 & 0 & N1; V1; C1 \\
\hline & & & & & & 7.286 & $\mathbf{0}$ & \\
\hline \multirow{2}{*}{ 09/05/2002 } & Areão & $578 m^{2}$ & $17: 10$ & 9 & 0 & 24 & 0 & N1; V1; C1 Foi capturado \\
\hline & & & & & & 3.429 & $\mathbf{0}$ & 01 adulto dia 08/05. \\
\hline \multirow{2}{*}{$16 / 05 / 2002$} & Areão & $578 m^{2}$ & $16: 55$ & 8 & 0 & 30 & 0 & N4; V1; C1 \\
\hline & & & & & & 4.286 & $\mathbf{0}$ & \\
\hline \multirow[t]{2}{*}{$23 / 05 / 2002$} & Areão & $578 m^{2}$ & 17:05 & 0 & 0 & 18 & 0 & N1; V3; C1 \\
\hline & & & & & & 2.571 & $\mathbf{0}$ & \\
\hline \multirow[t]{2}{*}{$29 / 05 / 2002$} & Areão & $578 m^{2}$ & $17: 25$ & 2 & 0 & 25 & 0 & N2; V1; C1 Nestas três \\
\hline & & & & & & 4.157 & $\mathbf{0}$ & $\begin{array}{l}\text { semanas anteriores } 04 \\
\text { animais adultos foram } \\
\text { mortos por cães. }\end{array}$ \\
\hline \multirow[t]{2}{*}{ 06/06/2002 } & Areão & $578 m^{2}$ & $17: 15$ & 6 & 3 & 20 & 0 & N2; V1; C1 \\
\hline & & & & & & 2.5 & $\mathbf{0}$ & \\
\hline \multirow[t]{2}{*}{$13 / 06 / 2002$} & Areão & $578 m^{2}$ & $17: 10$ & 8 & 0 & 23 & 0 & N1; V1; C1 \\
\hline & & & & & & 3.286 & $\mathbf{0}$ & \\
\hline $21 / 06 / 2002$ & Areão & $578 m^{2}$ & $17: 30$ & 5 & 0 & 40 & 0 & N1; V1; C1 \\
\hline & & & & & & 5 & $\mathbf{0}$ & \\
\hline 27/06/02 & Areão & $578 m^{2}$ & $17: 10$ & 1 & 0 & 40 & 0 & N2; V1; C1 \\
\hline & & & & & & 6.667 & $\mathbf{0}$ & \\
\hline 04/07/2002 & Areão & $578 m^{2}$ & $17: 10$ & 1 & 0 & 37 & 0 & N3; V2; C1 \\
\hline & & & & & & 5.286 & $\mathbf{0}$ & \\
\hline $11 / 07 / 02$ & Areão & $578 \mathrm{~m}^{2}$ & $17: 00$ & 3 & 0 & 54 & 0 & N4; V1; C2 \\
\hline & & & & & & 7.71 & $\mathbf{0}$ & \\
\hline 23/07/02 & Areão & $578 m^{2}$ & $17: 00$ & 0 & 0 & 31 & 0 & N1; V1; C1 \\
\hline & & & & & & 6.2 & $\mathbf{0}$ & \\
\hline $31 / 07 / 02$ & Areão & $578 m^{2}$ & $16: 40$ & 4 & 0 & 27 & 0 & N4; V2; C2 \\
\hline & & & & & & 4.5 & $\mathbf{0}$ & \\
\hline 07/08/2002 & Areão & $578 m^{2}$ & $16: 50$ & 10 & 0 & 38 & 0 & N1; V1; C1 \\
\hline & & & & & & 5.429 & $\mathbf{0}$ & \\
\hline $13 / 08 / 2002$ & Areão & $578 m^{2}$ & $16: 50$ & 10 & 0 & 13 & 0 & N3; V1; C1. 01 jovem \\
\hline & & & & & & 2.167 & $\mathbf{0}$ & \\
\hline 20/08/2002 & Areão & $578 m^{2}$ & $16: 30$ & 13 & 13 & 22 & 0 & N2; V2; C1. 05 jovens \\
\hline & & & & & & 3.143 & $\mathbf{0}$ & \\
\hline 28/08/2002 & Areão & $578 m^{2}$ & $17: 20$ & 14 & 13 & 20 & 0 & N1; V1; C1. 04 jovens \\
\hline & & & & & & 2.5 & $\mathbf{0}$ & \\
\hline $01 / 03 / 2002$ & Caterpilar & $384 m^{2}$ & $17: 50$ & 12 & 0 & Limp. & Limp. & N3; V1; C1 \\
\hline $07 / 03 / 2002$ & Caterpilar & $384 m^{2}$ & $18: 00$ & 19 & 0 & 3 & 0 & N3; V1; C1 \\
\hline & & & & & & 0.5 & $\mathbf{0}$ & \\
\hline $14 / 03 / 2002$ & Caterpilar & $384 m^{2}$ & 18:00 & 12 & 0 & 10 & 0 & N2; V1; C1 \\
\hline & & & & & & 1.429 & $\mathbf{0}$ & \\
\hline $21 / 03 / 2002$ & Caterpilar & $384 m^{2}$ & $18: 00$ & 21 & 0 & 15 & 0 & N1; V3; C1 \\
\hline & & & & & & 2.143 & $\mathbf{0}$ & \\
\hline 05/04/2002 & Caterpilar & $384 m^{2}$ & $17: 45$ & 7 & 0 & 34 & 0 & N1; V1; C1 \\
\hline & & & & & & 2.267 & $\mathbf{0}$ & \\
\hline $12 / 04 / 2002$ & Caterpilar & $384 m^{2}$ & $17: 35$ & 39 & 0 & 19 & 1 & N1; V1; C1 \\
\hline & & & & & & 2.714 & 0.143 & \\
\hline $16 / 04 / 2002$ & Caterpilar & $384 m^{2}$ & $17: 50$ & 24 & 3 & 9 & 0 & N1; V1; C1 \\
\hline & & & & & & 2.25 & $\mathbf{0}$ & \\
\hline $24 / 04 / 2002$ & Caterpilar & $384 m^{2}$ & $17: 40$ & 32 & 2 & 18 & 0 & N1; V1; C1 \\
\hline
\end{tabular}


Tabela 1. Planilha de campo

\begin{tabular}{|c|c|c|c|c|c|c|c|c|}
\hline \multirow[t]{2}{*}{ Dia } & \multirow[t]{2}{*}{ Local } & \multirow[t]{2}{*}{$\begin{array}{l}\text { Área } \\
\text { Amostral }\end{array}$} & \multirow[t]{2}{*}{ Hora } & \multicolumn{2}{|c|}{ Contagem Direta } & \multicolumn{2}{|c|}{$\begin{array}{c}\text { Contagem de Fezes } \\
\text { Fezes/Dia } \\
\end{array}$} & \multirow{2}{*}{$\begin{array}{l}\text { Observações: A-Adultos } \\
\\
\text { Tempo* }\end{array}$} \\
\hline & & & & Adulto & Filhote & Adulto & Filhote & \\
\hline \multirow[t]{2}{*}{$03 / 05 / 2002$} & Caterpilar & $384 m^{2}$ & $17: 15$ & 43 & 0 & 27 & 0 & N4; V1; C1 Aparentemente \\
\hline & & & & & & 3 & $\mathbf{0}$ & $\begin{array}{l}\text { está havendo intensa } \\
\text { dispersão de subadultos do } \\
\text { grupo principal. }\end{array}$ \\
\hline \multirow[t]{2}{*}{$10 / 05 / 2002$} & Caterpilar & $384 m^{2}$ & $17: 05$ & 3 & 0 & 14 & 0 & N1; V1; C1 \\
\hline & & & & & & 2 & $\mathbf{0}$ & \\
\hline \multirow[t]{2}{*}{$17 / 05 / 2002$} & Caterpilar & $384 m^{2}$ & $17: 00$ & 30 & 2 & 20 & 0 & N1; V1; C1 \\
\hline & & & & & & 2.857 & $\mathbf{0}$ & \\
\hline \multirow[t]{2}{*}{$22 / 05 / 2002$} & Caterpilar & $384 m^{2}$ & $17: 10$ & 35 & 0 & 13 & 0 & N4; V2; C1 \\
\hline & & & & & & 2.6 & $\mathbf{0}$ & \\
\hline \multirow{2}{*}{$30 / 05 / 2002$} & Caterpilar & $384 m^{2}$ & $16: 55$ & 42 & 4 & 19 & 0 & N1; V1; C1 \\
\hline & & & & & & 2.375 & $\mathbf{0}$ & \\
\hline \multirow[t]{2}{*}{ 07/06/2002 } & Caterpilar & $384 m^{2}$ & $16: 55$ & 15 & 7 & 19 & 1 & N1; V1; C1 \\
\hline & & & & & & 2.375 & 0.125 & \\
\hline \multirow[t]{2}{*}{$14 / 06 / 2002$} & Caterpilar & $384 m^{2}$ & $16: 55$ & 35 & 5 & 22 & 0 & N1; V1; C1 \\
\hline & & & & & & 3.143 & $\mathbf{0}$ & \\
\hline \multirow[t]{2}{*}{ 20/06/2002 } & Caterpilar & $384 m^{2}$ & $16: 40$ & 19 & 5 & 28 & 0 & N1; V1; C1 \\
\hline & & & & & & 4.667 & $\mathbf{0}$ & \\
\hline \multirow[t]{2}{*}{$28 / 06 / 02$} & Caterpilar & $384 m^{2}$ & $16: 00$ & 9 & 2 & 24 & 0 & N1; V2; C1 \\
\hline & & & & & & 3 & $\mathbf{0}$ & \\
\hline \multirow[t]{2}{*}{ 05/07/2002 } & Caterpilar & $384 m^{2}$ & $17: 20$ & 12 & 5 & 26 & 0 & N3; V1; C1 \\
\hline & & & & & & 3.714 & $\mathbf{0}$ & \\
\hline \multirow[t]{2}{*}{$12 / 07 / 02$} & Caterpilar & $384 m^{2}$ & $17: 00$ & 29 & 9 & 15 & 0 & N4; V2; C1 \\
\hline & & & & & & 2.14 & 0 & \\
\hline \multirow{2}{*}{$26 / 07 / 02$} & Caterpilar & $384 m^{2}$ & $17: 00$ & 5 & 3 & 18 & 0 & N2; V1; C1 \\
\hline & & & & & & 2.57 & $\mathbf{0}$ & \\
\hline \multirow[t]{2}{*}{ 02/08/02 } & Caterpilar & $384 m^{2}$ & $16: 50$ & 15 & 3 & 12 & 0 & N2; V2; C1 \\
\hline & & & & & & 1.71 & $\mathbf{0}$ & \\
\hline \multirow[t]{2}{*}{ 08/08/2002 } & Caterpilar & $384 m^{2}$ & $16: 45$ & 18 & 1 & 16 & 0 & N1; V1; C1 \\
\hline & & & & & & 2.667 & $\mathbf{0}$ & \\
\hline \multirow[t]{2}{*}{ 16/08/2002 } & Caterpilar & $384 m^{2}$ & $16: 30$ & 32 & 4 & 13 & 0 & N1; V1; C1 \\
\hline & & & & & & 1.625 & 0 & \\
\hline \multirow[t]{2}{*}{$23 / 08 / 2002$} & Caterpilar & $384 m^{2}$ & $16: 50$ & 45 & 4 & 11 & 0 & N1; V1; C1 \\
\hline & & & & & & 1.571 & $\mathbf{0}$ & \\
\hline \multirow[t]{2}{*}{$30 / 08 / 2002$} & Caterpilar & $384 m^{2}$ & $17: 00$ & 50 & 3 & 19 & $\mathbf{0}$ & N4; V1; C1 \\
\hline & & & & & & 2.714 & 0 & \\
\hline
\end{tabular}

*Vento (V): 1=sem; 2=fraco; 3=forte

Nebulosidade (N): $1=0<25 \%$; $2=25<50 \%$; $3=50<75 \%$; $4=75<100 \%$

Chuva (C): $1=$ sem; $2=$ fraca; $3=$ forte 